\title{
ON A GENERIC VERMA MODULE AT THE CRITICAL LEVEL OVER AFFINE LIE SUPERALGEBRAS
}

\author{
MARIA GORELIK
}

\begin{abstract}
We describe the structure of a Verma module with a generic highest weight at the critical level over a symmetrizable affine Lie superalgebra $\hat{\mathfrak{g}} \neq A(2 k, 2 l)^{(4)}$. We obtain the character formula for a simple module with a generic highest weight at the critical level conjectured by V. G. Kac and D. A. Kazhdan.
\end{abstract}

\section{INTRODUCTION}

It is well-known that the representation theory of a complex affine Lie algebra changes drastically at the critical level. In particular, Verma modules contain infinite number of singular vectors of imaginary degrees. As it is shown in $[\mathrm{Ku}$, a Verma module with a "generic" highest weight at the critical level looks like a polynomial algebra in a countable number of variables: submodules correspond to the ideals in the polynomial algebra and the Jantzen filtration corresponds to the adic filtration. Here "genericity" means that all singular vectors lie in the imaginary degrees. As a consequence, J.-M. Ku obtains the character formula conjectured by V. G. Kac and D. A. Kazhdan in [KK]: $\operatorname{ch} L(\lambda)=e^{\lambda} \prod_{\alpha \in \hat{\Delta}_{r e}^{+}}\left(1-e^{-\alpha}\right)^{-1}$, where $\lambda$ is a generic highest weight at the critical level and $L(\lambda)$ is the corresponding simple module. In this paper we extend the results of [Ku] to symmetrizable affine Lie superalgebras $\hat{\mathfrak{g}} \neq A(2 k, 2 l)^{(4)}$ (see 1.3).

For non-twisted affine Lie algebras the Kac-Kazhdan character formula was proven by different methods: for $\hat{\mathfrak{s} l}(2)$ by M. Wakimoto [Wk1], N. Wallach [W]; for the affinizations of classical algebras by T. Hayashi $[\mathrm{H}]$ and R. Goodman, N. Wallach [GW]; for the affinization of a general simple Lie algebra by B. Feigin and E. Frenkel $[\mathrm{FF}],[\mathrm{F}]$ and recently by T. Arakawa [Ar]; in finite characteristic by O. Mathieu [M]. For an arbitrary affine Lie algebras (including the twisted case) the formula was proven by J.-M. Ku $[\mathrm{Ku}]$ and recently reproven by M. Szczesny [Sz].

The approach of B. Feigin, E. Frenkel and M. Szczesny is based on the explicit realization of $L(\lambda)$ : they show that if $\lambda$ is a generic highest weight at the critical level then $L(\lambda)$ is isomorphic to a Wakimoto module, which is a representation of $\hat{\mathfrak{g}}$ in a Fock module over some infinite-dimensional Heisenberg algebra; the construction of Wakimoto modules uses a technique of vertex algebras. The Heisenberg algebra here corresponds to the set of real

1991 Mathematics Subject Classification. 17B67.

The author was partially supported by TMR Grant No. FMRX-CT97-0100. 
roots of $\hat{\mathfrak{g}}$. The method of J.-M. Ku is much more "elementary": it is based on a study of singular vectors in the Verma module $M(\lambda)$. It can be interpreted (see 1.2) in terms of an infinite-dimensional Heisenberg algebra which corresponds to the set of imaginary roots of $\hat{\mathfrak{g}}$ (this Heisenberg algebra is a subalgebra of $\hat{\mathfrak{g}}$ ). Our approach is close to one of J.-M. Ku.

1.1. Main result. Let $\hat{\mathfrak{g}}=\hat{\mathfrak{n}}_{-} \oplus \hat{\mathfrak{h}} \oplus \hat{\mathfrak{n}}$ be an affine Lie superalgebra with a symmetrizable indecomposable Cartan matrix (see 1.3). Let $M(\lambda)$ be a Verma module of the highest weight $\lambda$ and $v_{\lambda}$ be its canonical generator, which we assume to be even.

The Lie superalgebra $\hat{\mathfrak{n}}_{-}$admits a triangular decomposition $\hat{\mathfrak{n}}_{-}=\mathcal{N}_{-}^{-} \oplus \mathcal{H}_{-} \oplus \mathcal{N}_{-}^{+}$, where $\mathcal{H}_{-}$consists of the elements of imaginary weights (for a non-twisted case, $\mathcal{H}_{-}=\mathcal{L} \mathfrak{h} \cap \hat{\mathfrak{n}}_{-}$ and $\mathcal{N}_{-}^{ \pm}=\mathcal{L} \mathfrak{n}_{ \pm} \cap \hat{\mathfrak{n}}_{-}$, where $\mathcal{L}$ stands for the loop space of a given subalgebra of $\left.\mathfrak{g}\right)$. Set

$$
\mathcal{S}:=\mathcal{U}\left(\mathcal{H}_{-}\right) \text {. }
$$

Introduce the projections $\mathrm{HC}_{ \pm}: \mathcal{U}\left(\hat{\mathfrak{n}}_{-}\right) \rightarrow \mathcal{S}$, where

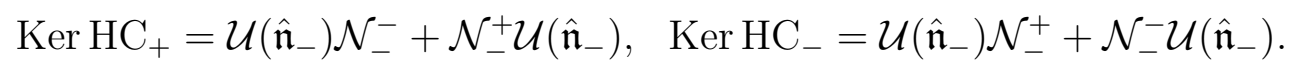

For each $\lambda \in \hat{\mathfrak{h}}^{*}$ define $\mathrm{HC}_{ \pm}: M(\lambda) \rightarrow \mathcal{S}$ via the natural identification of $M(\lambda)$ with $\mathcal{U}\left(\hat{\mathfrak{n}}_{-}\right)$; these projections play a central role in our description of $M(\lambda)$. The projection $\mathrm{HC}_{+}$appeared in $[\mathrm{Ku}]$ and $[\mathrm{Ch}]$.

We call $v \in M(\lambda)$ singular if $v$ is a weight vector and $v \in M(\lambda)^{\hat{\mathfrak{n}}}$ (these vectors are also called primitive). We say that $\lambda$ is a critical weight or $\lambda$ has the critical level if $(\lambda+\hat{\rho}, \delta)=0$ for an imaginary root $\delta$. If $\lambda$ is a critical weight then $M(\lambda)_{\lambda-\delta}$ contains a singular vector; we say that $\lambda$ is a generic critical weight $\left(\lambda \in \Lambda_{\text {crit }}\right)$ if $M(\lambda)_{\lambda-\alpha}$ has no singular vectors unless $\alpha$ is proportional to $\delta$.

If $\lambda$ is a generic critical weight, the space $M(\lambda)^{\hat{\mathfrak{n}}}$ has a natural structure of an associative (super)algebra. Indeed, the map $\psi \mapsto \psi\left(v_{\lambda}\right)$ gives an embedding of $\operatorname{End}_{[\hat{\mathfrak{g}}, \hat{\mathfrak{g}}]}(M(\lambda))$ into the space of singular vectors $M(\lambda)^{\hat{\mathfrak{n}}}$. The genericity condition on $\lambda$ means that this embedding is bijective and this provides $M(\lambda)^{\hat{\mathfrak{n}}}$ with the algebra structure.

In 1.1 .1 1.1.3 we assume that $\hat{\mathfrak{g}}$ is not of type $A(2 k, 2 l)^{(4)}$ and that $\lambda$ is a generic critical weight.

\subsubsection{Theorem. Let $\lambda$ be a generic critical weight.}

(i) One has $[M(\lambda): L(\lambda-s \delta)]=\operatorname{dim} M(\lambda)_{\lambda-s \delta}^{\hat{\mathfrak{n}}}$; thus any submodule of $M(\lambda)$ is generated by singular vectors.

(ii) The restrictions of $\mathrm{HC}_{+}: M(\lambda) \rightarrow \mathcal{S}$ and of $\mathrm{HC}_{-}: M(\lambda) \rightarrow \mathcal{S}$ to the space of singular vectors $M(\lambda)^{\hat{\mathfrak{n}}}$ give algebra isomorphisms $M(\lambda)^{\hat{\mathfrak{n}}} \stackrel{\sim}{\longrightarrow} \mathcal{S}$, where the image of $v_{\lambda}$ is $1 \in \mathcal{S}$.

(iii) Any singular vector generates a submodule isomorphic to $M(\lambda-s \delta)$ for some $s \geq 0$. 
Notice that $\mathcal{H}_{-}$is even and commutative so $\mathcal{S}$ is the algebra of polynomials in countably many variables.

1.1.2. For a submodule $N$ of $M(\lambda)$ set

$$
H(N):=\mathrm{HC}_{+}\left(N^{\hat{\mathfrak{n}}}\right) \subset \mathcal{S} .
$$

From Theorem 1.1.1 we see that $H$ provides a one-to-one correspondence between the submodules of $M(\lambda)$ and ad $\hat{\mathfrak{h}}$-invariant ideals of $\mathcal{S}$.

If $\lambda$ is a generic critical weight then all simple subquotients of $M(\lambda)$ are of the form $L(\lambda-s \delta)$; note that $L(\lambda) \cong L(\lambda-s \delta)$ as $[\hat{\mathfrak{g}}, \hat{\mathfrak{g}}]$-modules so $\operatorname{ch} L(\lambda-s \delta)=e^{-s \delta} \operatorname{ch} L(\lambda)$. As a result, the characters of $N$ and of $H(N)$ are connected by the following formula:

$$
\operatorname{ch} N=\operatorname{ch} L(\lambda) \cdot \operatorname{ch} H(N) \text {. }
$$

Applying this formula to $N=M(\lambda)$ we get the Kac-Kazhdan character formula:

$$
\operatorname{ch} M(\lambda)=\operatorname{ch} L(\lambda) \cdot \operatorname{ch} \mathcal{S},
$$

that is

$$
\operatorname{ch} L(\lambda)=e^{\lambda} \prod_{\alpha \in \hat{\Delta}_{r e ; \overline{0}}^{+}}\left(1-e^{-\alpha}\right)^{-1} \prod_{\alpha \in \hat{\Delta}_{r e ; \bar{T}}^{+}}\left(1+e^{-\alpha}\right) .
$$

Note that $\operatorname{ch} L(\lambda)=e^{\lambda} \operatorname{ch} \mathcal{U}\left(\mathcal{N}_{-}^{+}\right) \operatorname{ch} \mathcal{U}\left(\mathcal{N}_{-}^{-}\right)$

1.1.3. Jantzen filtration. Recall that $\mathcal{H}_{-}$is commutative so $\mathcal{S}=\mathcal{U}\left(\mathcal{H}_{-}\right)$is the symmetric algebra: $\mathcal{S}=\sum_{j=0}^{\infty} \mathcal{S}^{j}$. The spaces $\mathcal{S}^{\geq k}:=\sum_{j=k}^{\infty} \mathcal{S}^{j}$ form the adic filtration on $\mathcal{S}$.

Theorem. If $\lambda$ is a generic critical weight then $H$ maps the Jantzen filtration $\left\{M(\lambda)^{k}\right\}$ to the adic filtration on $\mathcal{S}$, i.e. $H\left(M(\lambda)^{k}\right)=\mathcal{S}^{\geq k}$.

As a result, the Jantzen filtration coincides with the cosocle filtration.

Combining Theorems 1.1.1, 1.1.3 we obtain the

Corollary. $M(\lambda)^{k}$ is generated by the singular vectors $\mathrm{HC}_{+}^{-1}\left(\mathcal{S}^{k}\right)$.

1.2. Connection with representations of Heisenberg algebra. Let $\mathcal{H}$ (resp., $\mathcal{H}_{-}$) be the sum of positive (resp., negative) imaginary root spaces of $\hat{\mathfrak{g}}$. Set $\mathfrak{l}=\mathcal{H}_{-} \oplus \mathbb{C} K \oplus \mathbb{C} D \oplus \mathcal{H}$ and let $V^{k}$ be the induced module $V^{k}=\operatorname{Ind}_{\mathbb{C} K \oplus \mathbb{C} D \oplus \mathcal{H}}^{\mathfrak{l}} \mathbb{C}_{k}$ from the one-dimensional module $\mathbb{C}_{k}$ with trivial action of $\mathcal{H}+\mathbb{C} D$ and $K=k \in \mathbb{C}$. Identify $V^{k}$ with $\mathcal{S}=\mathcal{U}\left(\mathcal{H}_{-}\right)$and say that $v \in V^{k}$ is singular if $\mathcal{H} v=0$ and $v$ is an $D$-eigenvector.

Let $\hat{\mathfrak{g}} \neq A(2 k, 2 l)^{(4)}$. Then $\mathcal{H}_{-} \oplus \mathbb{C} K \oplus \mathcal{H}$ is a countably dimensional Heisenberg algebra and $V^{k}$ is irreducible if and only if $k \neq 0$. In $V^{0}$ any $D$-eigenvector is singular; the Jantzen filtration of $V^{0}$ identifies with the adic filtration of $\mathcal{S}$. Theorems 1.1.1]1.1.3 can be reformulated in the following way. 
1.2.1. $M(\lambda)$ with a generic highest weight looks like $V^{k}$ for $k:=(\lambda+\hat{\rho}, \delta)$. More precisely, $\mathrm{HC}_{+}: M(\lambda) \rightarrow \mathcal{S}$ maps singular vectors to the singular ones and induces a bijection between the submodules of $M(\lambda)$ and of $V^{k}$. This bijection is compatible with the Jantzen filtrations.

1.2.2. In [GS the result similar to 1.2.1 is proven for a non-symmetrizable affine Lie superalgebra $q(n)^{(2)}$. In this case $V^{k}$ is reducible for all $k \in \mathbb{C}$ and $M(\lambda)$ is reducible for all $\lambda \in \hat{\mathfrak{h}}^{*}$.

1.2.3. We believe that a result similar to 1.2 .1 holds for $\hat{\mathfrak{g}}=A(2 k, 2 l)^{(4)}$. A difficulty in this case is that $\mathcal{H}_{-}$is not commutative and it is not true that any submodule of $V^{k}$ (and of $M(\lambda))$ is generated by singular vectors.

1.3. Affine Lie superalgebras. Recall that affine Lie algebras are finite growth KacMoody algebras. The Cartan matrix of an affine Lie algebra is symmetrizable [K2]. An affine Lie algebra can be described in terms of a finite-dimensional semisimple Lie algebra and its finite order automorphism (see, for example, [K2], Ch. VI-VIII).

The superalgebra generalization of Kac-Moody algebras was introduced in [K1]; a detailed treatment of this notion can be found in [Wk2]. Call a Kac-Moody superalgebra affine if it has a finite growth and symmetrizable if it has a symmetrizable Cartan matrix. In $\mathrm{vdL}$, [S] the affine symmetrizable Lie superalgebras are described in terms of finitedimensional Kac-Moody superalgebras and their finite order automorphisms; we recall this construction in 2.3.

A symmetrizable affine Lie superalgebra has a Casimir element. As a consequence, a Verma module $M(\lambda)$ is irreducible unless $\lambda$ belongs to the union of countably many hyperplanes. Among these hyperplanes one is rather special: $M(\lambda)$ has an infinite length if $\lambda$ lies on this hyperplane. This hyperplane is the set of critical weights.

Affine Lie superalgebras were classified in a recent paper [HS]. It turns out that nonsymmetrizable affine Lie superalgebras consist of 4 series. One of this series is $q(n)^{(2)}$; these algebras are twisted affinizations of "strange" Lie superalgebras $q(n)$. As it is mentioned above, a Verma module over $q(n)^{(2)}$ is always reducible. It is an interesting question to study Verma modules of generic highest weight for other series.

1.4. Outline of the proof. In 1.4.1, 1.4.2 below we outline the proof of theorems 1.1.1, 1.1.3. Retain notation of 1.1.

1.4.1. In 3.2 we show that theorems 1.1.1, 1.1.3 can be easily deduced from the following assertions for the Verma module of a generic critical weight $\lambda$ :

(A) the restrictions of $\mathrm{HC}_{ \pm}$to the set of singular vectors of $M(\lambda)^{k}$ contain $\mathcal{S}^{\geq k}$,

(B) $\left[M(\lambda)^{k}: L(\lambda-s \delta)\right]=\operatorname{dim} \mathcal{S}_{-s \delta}^{\geq k}$ for all $k, s$. 
We prove (A), (B) as follows. Let $A$ be the local algebra $A=\mathbb{C}[x]_{(x)}$ and $M(\lambda+x \xi)$ be a Verma module over $\hat{\mathfrak{g}}_{A}=\hat{\mathfrak{g}} \otimes A$. Recall that the Jantzen filtration $M(\lambda)^{k}$ is obtained by the evaluation of the Jantzen filtration $\mathcal{F}^{k}(M(\lambda+x \xi))$ at $x=0$.

Let $\sigma$ be the natural anti-involution of $\hat{\mathfrak{g}}$ (it interchanges the canonical generators $e_{i}, f_{i}$ and acts by id to $\hat{\mathfrak{h}})$. Set $\mathcal{N}^{ \pm}=\sigma\left(\mathcal{N}_{-}^{\mp}\right)$.

For $\lambda$ generic (this means that either $\lambda$ is a generic critical weight or $M(\lambda)$ is simple) we construct linear maps $\mathrm{HC}_{ \pm, A}^{-1}: \mathcal{S}_{A} \rightarrow M(\lambda+x \xi)^{\mathcal{N}^{ \pm}}$. Denote by $\mathrm{HC}_{ \pm}^{-1}: \mathcal{S} \rightarrow M(\lambda)$ the reductions of $\mathrm{HC}_{ \pm, A}^{-1}$ modulo $(x)$. We show that the images of $\mathrm{HC}_{ \pm}^{-1}$ lie in $M(\lambda)^{\hat{\mathfrak{n}}}$ if $\lambda$ is a generic critical weight.

Let $(-,-)$ be the Shapovalov form on $M(\lambda+x \xi)$. One readily sees that $M(\lambda+x \xi)^{\mathcal{N}^{+}}$is orthogonal to $\mathrm{Ker} \mathrm{HC}_{-}$with respect to this form. As a result, $\mathrm{HC}_{+, A}^{-1}(z) \in \mathcal{F}^{k}(M(\lambda+x \xi))$ if $\left(\mathrm{HC}_{+, A}^{-1}(z), \mathrm{HC}_{-, A}^{-1}(\mathcal{S})\right) \subset(x)^{k}$. We prove the last inclusion for $k$ equal to the degree of $z$ by induction on this degree; the proof is based on the fact that $\mathrm{HC}_{-}^{-1}\left(z^{\prime}\right)$ is singular for any $z^{\prime}$. This is the point where we use simultaneously both projections $\mathrm{HC}_{ \pm}$in order to prove a certain assertion for $\mathrm{HC}_{+}$. This gives $(\mathrm{A})$ for $\mathrm{HC}_{+}$; the proof for $\mathrm{HC}_{-}$is similar.

The proof of (B) is based on (A) and the formula:

$$
\sum_{k \geq 1} \operatorname{ch} M(\lambda)^{k}=\frac{\operatorname{ch} M(\lambda)}{\operatorname{ch} \mathcal{S}} \sum_{k \geq 1} \operatorname{ch} \mathcal{S}^{\geq k}
$$

which easily follows from the Jantzen sum formula. We deduce (B) from this formula by induction on weight.

1.4.2. One of the key points of the proof is the construction of $\mathrm{HC}_{+, A}^{-1}(z)$ for $z \in \mathcal{H}_{-}$and the proof that $\mathrm{HC}_{+}(z)$ is singular if $\lambda$ is a generic critical weight.

Let $\lambda$ be generic. The construction of $\mathrm{HC}_{+, A}^{-1}(z)$ for $z=a(-m) \in \mathcal{H}_{-}(m>0)$ follows the construction of singular vectors in $M(\lambda)$ given in [ $\mathrm{Ku}]$; we outline this construction in 6.2 .

The proof that $\mathrm{HC}_{+}^{-1}(z)$ is singular goes as follows. It is easy to see that for generic $\lambda$ a vector $v \in M(\lambda)_{\lambda-m \delta}$ is singular if $\left(\mathcal{N}^{+}+\mathcal{H}\right) v=0$. By the construction $v:=\mathrm{HC}_{+}^{-1}(z)$ is annihilated by $\mathcal{N}^{+}$and by the elements of $\mathcal{H}_{s \delta}$ for $s \neq m$. From 2.6.1 it is easy to deduce that for $u \in \mathcal{H}_{m}$ one has

$$
u v=(u \mid a(-m))(m K+c) v_{\lambda}
$$

where $c$ is a scalar which does not depend on $\lambda$. Therefore $v$ is singular if $\lambda$ has level $-c / m$. Recall that $M(\lambda)$ is simple if the level is not critical so $v$ is not singular in this case. Hence $v$ is singular if the level of $\lambda$ is critical.

1.5. Acknowledgment. I am very grateful to M. Duflo for fruitful discussions. A part of this work was done during my stay at IHES and at MPIM. I am grateful to these institutions for stimulating atmosphere and excellent working conditions. 


\section{Preliminaries And nOtation}

Our base field is $\mathbb{C}$; we denote by $\mathbb{Z}_{\geq 0}$ the set of non-negative integers. If $V$ is a superspace, we denote by $p(v)$ the parity of a vector $v \in V$. For a Lie superalgebra $\mathfrak{g}=\mathfrak{g}_{\overline{0}} \oplus \mathfrak{g}_{\overline{1}}$ we denote by $\mathcal{U}(\mathfrak{g})$ its universal enveloping algebra and by $\mathcal{S}(\mathfrak{g})$ its symmetric algebra. We view $\mathcal{U}(\mathfrak{g})$ as a $\mathfrak{g}$-module with respect to the adjoint action.

If $\mathfrak{p}$ is a Lie algebra, $N$ is a $\mathfrak{p}$-module and $N^{\prime}$ is a subspace of $N$, denote by $\mathfrak{p} N^{\prime}$ the vector space spanned by $x v$ where $x \in \mathfrak{p}, v \in N^{\prime}$.

2.1. Index of notations. Symbols used frequently are given below under the section number where they are first defined.

$\begin{array}{llll}2.5 & \tilde{\mathcal{H}}, \tilde{\mathcal{N}}^{ \pm}, & 2.7 .1 & \mathcal{H}, \mathcal{N}^{ \pm}, \mathcal{H}_{-}, \mathcal{N}_{-}^{ \pm} \\ 2.9 .1 & \mathcal{S}, \mathcal{S}^{k}, \mathcal{S}^{\geq k} & 2.9 .2 & \mathrm{HC}_{+}, \mathrm{HC}_{-}, \breve{M} \\ 2.11 & \Omega(M), \text { wt } u & 2.12 & \Lambda, \Lambda_{\text {crit }} .\end{array}$

2.2. Triangular decompositions of superalgebras. A triangular decomposition of a Lie superalgebra $\mathfrak{g}$ can be constructed as follows (see [PS]). A Cartan algebra is a nilpotent subalgebra which coincides with its normalizer. Fix a Cartan algebra $\mathfrak{h}$. Then $\mathfrak{g}$ has a generalized root decomposition $\mathfrak{g}:=\mathfrak{h}+\oplus_{\alpha \in \Delta} \mathfrak{g}_{\alpha}$, where $\Delta$ is a subset of $\mathfrak{h}^{*}$ and

$$
\mathfrak{g}_{\alpha}=\left\{x \in \mathfrak{g} \mid(\operatorname{ad}(h)-\alpha(h))^{\operatorname{dim} \mathfrak{g}}(x)=0\right\} .
$$

In this paper all Cartan algebras are pure even and any root space $\mathfrak{g}_{\alpha}$ is either odd or even. This allows one to define the parity on the set of roots $\Delta$ : we denote by $\Delta_{0}$ (resp. $\Delta_{1}$ ) the set of non-zero weights of $\mathfrak{g}_{\overline{0}}$ (resp., $\mathfrak{g}_{\overline{1}}$ ) with respect to $\mathfrak{h}$; one has $\Delta=\Delta_{0} \coprod \Delta_{1}$.

Now fix $h \in \mathfrak{h}_{0}$ satisfying $\operatorname{Re} \alpha(h) \neq 0$ for $\alpha(h) \neq 0$ for any $\alpha \in \Delta$ (i.e. $\alpha(h)$ is not a non-zero imaginary number). Set

$$
\begin{aligned}
& \Delta^{+}:=\{\alpha \in \Delta \mid \operatorname{Re} \alpha(h)>0\}, \\
& \mathfrak{n}:=\sum_{\alpha \in \Delta^{+}} \mathfrak{g}_{\alpha} .
\end{aligned}
$$

Define $\Delta^{-}$and $\mathfrak{n}_{-}$similarly. Then $\mathfrak{g}=\mathfrak{n}_{-} \oplus \mathfrak{h} \oplus \mathfrak{n}$ is a triangular decomposition.

2.3. Affine Lie superalgebras with symmetrizable Cartan matrices. According to vdL, any Kac-Moody superalgebra, which has finite Gelfand-Kirillov dimension and a symmetrizable Cartan matrix, can be described in terms of the loop algebra of a finite dimensional Kac-Moody Lie superalgebra and its automorphism of a finite order. The non-twisted affine Lie superalgebras correspond to the trivial automorphism. The twisted affine Lie superalgebras correspond to the Dynkin diagrams of types $X_{N}=A(2 k, 2 l-1)$, $A(2 k-1,2 l-1)((k, l) \neq(1,1)), A(2 k, 2 l), C(l+1), D(k+1, l), G_{3}$ and automorphisms of order $2,2,4,2,2,2$ respectively. We briefly recall the construction below. 
2.3.1. Let $X_{N}$ be a Dynkin diagram of a Kac-Moody finite dimensional Lie superalgebra $\mathfrak{g}=\mathfrak{n}_{-} \oplus \mathfrak{h} \oplus \mathfrak{n}$ with the triangular decomposition determined by this Dynkin diagram. We may (and will) assume that $X_{N}$ is connected. Then $\mathfrak{g}$ is either simple or of the type $\mathfrak{g l}(n \mid n)=A(n-1, n-1)$. The even part $\mathfrak{g}_{0}$ is reductive. The algebra $\mathfrak{g}$ admits a nondegenerate even invariant bilinear form $(-\mid-)^{\prime}$; if $\mathfrak{g}$ is of the type $A(m, n)$ we take the form $(a \mid b)^{\prime}=\operatorname{str} a b$.

2.3.2. The affine Lie superalgebra $\hat{\mathfrak{g}}^{\prime}$ corresponding to the Dynkin diagram $X_{N}^{(1)}$ can be described as follows: $\hat{\mathfrak{g}}^{\prime}=\oplus_{m \in \mathbb{Z}} \hat{\mathfrak{g}}_{m}^{\prime}$, where $\hat{\mathfrak{g}}_{0}^{\prime}=\mathfrak{g} \oplus \mathbb{C} K^{\prime} \oplus \mathbb{C} D^{\prime}$ and, for $m \neq 0, \hat{\mathfrak{g}}_{m}^{\prime}:=\mathfrak{g} t^{m}$ if $\mathfrak{g} \neq \mathfrak{g l}(n \mid n), \hat{\mathfrak{g}}_{m}^{\prime}:=\mathfrak{p} \mathfrak{g l}(n \mid n) t^{m}$ for $\mathfrak{g}=\mathfrak{g l}(n \mid n)$. The structure of superspace on $\hat{\mathfrak{g}}^{\prime}$ is given by $p(t)=p\left(K^{\prime}\right)=p\left(D^{\prime}\right)=0$ and the commutational relations are

$$
[a(m), b(k)]=[a, b](m+k)+m \delta_{m,-k}(a \mid b)^{\prime} K^{\prime}, \quad\left[\hat{\mathfrak{g}}, K^{\prime}\right]=0, \quad\left[D^{\prime}, a(m)\right]=m a(m),
$$

where $a(m):=a t^{m}$ for $a \in \mathfrak{g}, m \in \mathbb{Z}$. If $\mathfrak{g}=\mathfrak{g l}(m, m)$ then the term $[a, b]$ should be substituted by its image in $\mathfrak{p g l}(n \mid n)$ if $m+k \neq 0$ and $m k=0$.

The form $(-\mid-)^{\prime}$ can be extended from $\mathfrak{g} \subset \hat{\mathfrak{g}}_{0}^{\prime}$ to $\hat{\mathfrak{g}}^{\prime}$ by setting $\left(K^{\prime} \mid D^{\prime}\right)^{\prime}=0,\left(K^{\prime} \mid D^{\prime}\right)^{\prime}=1$ and $\left(x t^{m} \mid y t^{n}\right)^{\prime}=\delta_{m, n}(x \mid y)^{\prime},\left(\mathbb{C} K^{\prime}+\mathbb{C} D^{\prime} \mid x t^{m}\right)^{\prime}=0$ for any $x, y \in \mathfrak{g}$.

2.3.3. Let $\epsilon$ be an automorphism of $\mathfrak{g}$ of a finite order $r(r=1,2,3,4)$ such that $\epsilon(\mathfrak{h})=\mathfrak{h}$. Extend $\epsilon$ to $\hat{\mathfrak{g}}^{\prime}$ by putting $\epsilon(t)=\exp (-2 \pi i / r) t$, that is

$$
\epsilon\left(a t^{m}\right)=\exp (-2 \pi m i / r) \epsilon(a) t^{m}, \quad \epsilon\left(K^{\prime}\right)=K^{\prime}, \quad \epsilon\left(D^{\prime}\right)=D^{\prime} .
$$

By [K2], $\mathrm{vdL}$ the affine Lie superalgebra $\hat{\mathfrak{g}}$ corresponding to the Dynkin diagram $X_{N}^{(r)}$ is isomorphic to the subalgebra of invariants $\left(\hat{\mathfrak{g}}^{\prime}\right)^{\epsilon}$. We identify this subalgebras:

$$
\hat{\mathfrak{g}}:=\left(\hat{\mathfrak{g}}^{\prime}\right)^{\epsilon} \text {. }
$$

Clearly, $\hat{\mathfrak{g}}$ inherits the $\mathbb{Z}$-grading: $\hat{\mathfrak{g}}=\oplus_{m \in \mathbb{Z}} \hat{\mathfrak{g}}_{m}$, where $\hat{\mathfrak{g}}_{m}=\left(\hat{\mathfrak{g}}_{m}^{\prime}\right)^{\epsilon}$.

Introduce $K:=r K^{\prime}, D:=D^{\prime}$ and the form $(-\mid-):=(-\mid-)^{\prime} / r$. The restriction of $(-\mid-)$ gives an invariant form on $\hat{\mathfrak{g}}$. We identify $\mathfrak{g}^{\epsilon} \oplus \mathbb{C} K \oplus \mathbb{C} D$ with the zero homogeneous component $\hat{\mathfrak{g}}_{0}$.

2.3.4. Recall ([K2], 8,1) that any automorphism of a finite order of the reductive algebra $\mathfrak{g}_{\overline{0}}$ has an invariant regular element. Without loss of generality we choose the triangular decomposition $\mathfrak{g}_{\overline{0}}=\mathfrak{n}_{-, \overline{0}} \oplus \mathfrak{h} \oplus \mathfrak{n}_{\overline{0}}$ determined by this regular element.

Then $\mathfrak{h}^{\epsilon}$ is a Cartan subalgebra of $\mathfrak{g}^{\epsilon}$ and the centralizer of $\mathfrak{h}^{\epsilon}$ in $\mathfrak{g}_{0}$ coincides with $\mathfrak{h}$. We have a triangular decomposition $\mathfrak{g}_{0}^{\epsilon}=\mathfrak{n}_{-, \overline{0}}^{\epsilon} \oplus \mathfrak{h}^{\epsilon} \oplus \mathfrak{n} \frac{\epsilon}{\overline{0}}$. Recall that all triangular decompositions of $\mathfrak{g}_{0}^{\epsilon}$ are conjugate by inner automorphisms of $\mathfrak{g}_{0}^{\epsilon}$. Since any inner automorphism of $\mathfrak{g}_{0}^{\epsilon}$ can be extended to $\hat{\mathfrak{g}}$, any triangular decomposition

$$
\hat{\mathfrak{g}}=\hat{\mathfrak{n}}_{-} \oplus \hat{\mathfrak{h}} \oplus \hat{\mathfrak{n}}
$$


is conjugate by $\operatorname{Aut}(\hat{\mathfrak{g}})$ to a triangular decomposition compatible with $\mathfrak{g}_{0}^{\epsilon}=\mathfrak{n}_{-, \overline{0}}^{\epsilon} \oplus \mathfrak{h}^{\epsilon} \oplus \mathfrak{n}_{\overline{0}}^{\epsilon}$. Hence without loss of generality we may (and will) assume that

$$
\hat{\mathfrak{h}}=\mathfrak{h}^{\epsilon} \oplus \mathbb{C} K \oplus \mathbb{C} D, \quad \mathfrak{n}_{0}^{\epsilon} \subset \hat{\mathfrak{n}} .
$$

By above $\mathfrak{h}$ is the centralizer of $\mathfrak{h}^{\epsilon}$ in $\mathfrak{g}_{\overline{0}}$. If $\hat{\mathfrak{g}}$ is not of the type $A(2 k, 2 l)^{(4)}=\mathfrak{s l}(2 k+$ $1,2 l+1)^{(4)}$ the centralizer of $\mathfrak{h}^{\epsilon}$ in $\mathfrak{g}_{\overline{1}}$ is zero. For $A(2 k, 2 l)^{(4)}$ the centralizer of $\mathfrak{h}^{\epsilon}$ in $\mathfrak{g}_{\overline{1}}$ has dimension two. Hence

$$
\begin{aligned}
& \hat{\mathfrak{g}}^{\mathfrak{h}^{\epsilon}}=\hat{\mathfrak{h}}+\sum_{k \neq 0}\left(\mathfrak{h} t^{k}\right)^{\epsilon} \text {, for } \hat{\mathfrak{g}} \neq A(2 k, 2 l)^{(4)} \text {, } \\
& \hat{\mathfrak{g}}_{0}^{\mathfrak{h}^{\epsilon}}=\hat{\mathfrak{h}}+\sum_{k \neq 0}\left(\mathfrak{h} t^{k}\right)^{\epsilon} \text {, for } \hat{\mathfrak{g}}=A(2 k, 2 l)^{(4)} \text {. }
\end{aligned}
$$

2.4. Roots. Denote by $\Delta^{+}$(resp., $\hat{\Delta}^{+}$) the multiset of positive roots of $\mathfrak{g}$ (resp., of $\hat{\mathfrak{g}}$ ), and by $\hat{\Delta}_{0}^{+}$(resp., $\hat{\Delta}_{1}^{+}$) the multiset of even (resp., odd) positive roots. Remark that $\Delta^{+}$ is a usual set (all root spaces in $\mathfrak{g}$ are one-dimensional).

The bilinear form $(-\mid-)$ induces a non-degenerate bilinear form $(-,-)$ on $\hat{\mathfrak{h}}^{*}$. Let $h_{\alpha}$ be the image of $\alpha$ under the isomorphism $\hat{\mathfrak{h}}^{*} \rightarrow \hat{\mathfrak{h}}$ induced by the bilinear form (that is $\left.\mu\left(h_{\alpha}\right)=(\alpha, \mu)\right)$.

2.4.1. Real roots are defined via the action of Weyl group (see Wk2 for the definition) on $\hat{\Delta}^{+}$; the roots is imaginary if it is not real. For a symmetrizable affine Lie superalgebras a root $\alpha \in \hat{\Delta}^{+}$is imaginary if $(\alpha, \beta)=0$ for all $\beta \in \hat{\Delta}$. Denote by $\hat{\Delta}_{r e}^{+}$(resp., $\left.\hat{\Delta}_{i m}^{+}\right)$the multiset of real (resp., imaginary) positive roots; one has $\hat{\Delta}^{+}=\hat{\Delta}_{r e}^{+} \amalg \hat{\Delta}_{i m}^{+}$. All imaginary roots are proportional to a certain element $\delta \in \hat{\mathfrak{h}}^{*}:$ if $\hat{\mathfrak{g}}_{\alpha} \subset \hat{\mathfrak{g}}_{s}$ and $\alpha$ is imaginary then $\alpha=s \delta$. Notice that $h_{\delta}=K$, since $h_{\delta}$ is central and that $\left(D \mid h_{\delta}\right)=D(\delta)=1$.

Apart of the case $G(3)^{(2)}$ one has $\hat{\Delta}_{i m}^{+}=\mathbb{Z} \delta$; for $G(3)^{(2)}$ one has $\hat{\Delta}_{i m}^{+}=\mathbb{Z}(2 \delta)$, i.e. $\delta$ is not a root. All imaginary roots are even if $\hat{\mathfrak{g}}$ is not of the type $A(2 k, 2 l)^{(4)}$.

For a non-twisted case $\hat{\Delta}_{r e}^{+}=\mathbb{Z} \delta+\Delta, \mathfrak{g}_{\alpha+m \delta}=\mathfrak{g}_{\alpha} t^{m}$ and $\hat{\mathfrak{g}}_{m \delta}=\mathfrak{h} t^{m}$ for $m \neq 0$.

2.4.2. Let $\hat{\pi}$ be the set of simple roots for $\hat{\Delta}^{+}$. Let $\hat{\rho} \in \hat{\mathfrak{h}}$ be such that $(\hat{\rho}, \alpha)=\frac{1}{2}(\alpha, \alpha)$ for any $\alpha \in \hat{\pi}$. For a weight $\lambda \in \hat{\mathfrak{h}}^{*}$ the value $\lambda(K)$ is called a level. The weights of a given level form a hyperplane in $\hat{\mathfrak{h}}^{*}$. Put $h^{\vee}:=\hat{\rho}(K)$. The level $-h^{\vee}$ is called critical: $\lambda$ has the critical level iff $(\lambda+\hat{\rho}, \delta)=0$.

2.4.3. Set $\hat{Q}^{+}:=\sum_{\alpha \in \hat{\Delta}^{+}} \mathbb{Z}_{\geq 0} \alpha$. Define a partial ordering on $\hat{\mathfrak{h}}^{*}$ by setting $\mu \geq \mu^{\prime}$ if $\mu-\mu^{\prime} \in \hat{Q}^{+}$. 
2.5. Algebras $\mathcal{H}, \mathcal{N}^{ \pm}$. One has $\hat{\mathfrak{g}}=[\hat{\mathfrak{g}}, \hat{\mathfrak{g}}] \oplus \mathbb{C} D$. Retain notation of 2.3 .3 and set

$$
\mathfrak{h}^{\prime}:=\hat{\mathfrak{h}} \cap[\hat{\mathfrak{g}}, \hat{\mathfrak{g}}] \text {. }
$$

One has $\mathfrak{h}^{\prime}=\mathfrak{h}^{\epsilon}+\mathbb{C} K$. Observe that the decomposition $[\hat{\mathfrak{g}}, \hat{\mathfrak{g}}]=\hat{\mathfrak{n}}_{-} \oplus \mathfrak{h}^{\prime} \oplus \hat{\mathfrak{n}}$ is not triangular in a sense of 2.2, since the centralizer of $\mathfrak{h}^{\prime}$ is

$$
\tilde{\mathcal{H}}:=\mathfrak{h}^{\prime}+\sum_{j \neq 0} \hat{\mathfrak{g}}_{j \delta} .
$$

Fix $h^{\prime} \in \mathfrak{h}^{\prime}$ in such a way that $\operatorname{Re} \alpha\left(h^{\prime}\right)>0$ if $\alpha$ is a weight of $\hat{\mathfrak{n}} \cap \hat{\mathfrak{g}}_{0}$ and that $\operatorname{Re} \alpha\left(h^{\prime}\right) \neq 0$ for any $\alpha \in \hat{\Delta}_{r e}$. Fix a triangular decomposition

$$
[\hat{\mathfrak{g}}, \hat{\mathfrak{g}}]=\tilde{\mathcal{N}}^{+} \oplus \tilde{\mathcal{H}} \oplus \tilde{\mathcal{N}}^{-}
$$

defined via $h^{\prime}$ as in 2.2. Then $\hat{\mathfrak{n}} \cap \hat{\mathfrak{g}}_{0} \subset \tilde{\mathcal{N}}^{+}, \hat{\mathfrak{n}}_{-} \cap \hat{\mathfrak{g}}_{0} \subset \tilde{\mathcal{N}}^{-}$. If $\hat{\mathfrak{g}}$ is a non-twisted affinization one has $\tilde{\mathcal{N}}^{ \pm}=\sum_{m \in \mathbb{Z}} \mathfrak{n}_{ \pm} t^{m}$.

Let $\hat{\mathfrak{g}}$ is not of the type $A(2 k, 2 l)^{(4)}$. By (2),$\tilde{\mathcal{H}}$ is spanned elements of the form $h t^{k}, h \in \mathfrak{h}$ and by $K$. In particular, $\tilde{\mathcal{H}}$ is isomorphic to the direct product of countably dimensional Heisenberg algebra $\mathbb{C} K+\sum_{j \neq 0} \hat{\mathfrak{g}}_{j \delta}$ and the abelian Lie algebra $\mathfrak{h}^{\epsilon}$.

2.6. Automorphism of $\hat{\mathfrak{g}}$. In Lemma 2.6 .2 we will show that for each $\hat{\mathfrak{g}}$ there exist a commuting pair $\epsilon, \phi \in \operatorname{Aut}(\mathfrak{g})$ such that $\hat{\mathfrak{g}}=\left(\hat{\mathfrak{g}}^{\prime}\right)^{\epsilon}$ in a sense of 2.3 .3 and $\left.\phi\right|_{\mathfrak{h}}=-\mathrm{id}$.

Since $\left.\phi\right|_{\mathfrak{h}}=-\mathrm{id}$, the bilinear form $(-,-)$ is $\phi$-invariant and this allows us to define an automorphism $\hat{\phi}$ of $\hat{\mathfrak{g}}^{\prime}$ by the formulas:

$$
\hat{\phi}\left(x t^{k}\right)=\phi(x) t^{k}, \quad \hat{\phi}(K)=K, \quad \hat{\phi}(D)=D .
$$

Since $\phi$ commutes with $\epsilon$, the automorphism $\hat{\phi}$ stabilizes $\hat{\mathfrak{g}}$. View the restriction of $\hat{\phi}$ to $\hat{\mathfrak{g}}$ as an automorphism of $\hat{\mathfrak{g}}$. Set

$$
(\mathbb{C} K+\mathbb{C} D)^{\perp}:=\{h \in \hat{\mathfrak{h}} \mid(h, K)=(h, D)=0\} .
$$

2.6.1. Corollary. $\hat{\phi} \in \operatorname{Aut}(\hat{\mathfrak{g}})$ has the following properties

(i) $\hat{\phi}(K)=K, \quad \hat{\phi}(D)=D,\left.\quad \hat{\phi}\right|_{(\mathbb{C} K+\mathbb{C} D)^{\perp}}=-\mathrm{id} ;$

(ii) $\quad$ for $\hat{\mathfrak{g}} \neq\left. A(2 k, 2 l)^{(4)} \hat{\phi}\right|_{\tilde{\mathcal{H}}_{j}}=-\mathrm{id}$, for $j \neq 0$;

$\left(i i^{\prime}\right) \quad$ for $\hat{\mathfrak{g}}=\left.A(2 k, 2 l)^{(4)} \quad \hat{\phi}\right|_{\tilde{\mathcal{H}}_{2 j}}=-\mathrm{id}, \quad$ for $j \neq 0,\left.\quad \hat{\phi}\right|_{\tilde{\mathcal{H}}_{4 k \pm 1}}= \pm \sqrt{-1} \mathrm{id}$;

(iii) $\hat{\phi}\left(\tilde{\mathcal{N}}_{k}^{+}\right)=\tilde{\mathcal{N}}_{k}^{-}, \quad \hat{\phi}\left(\tilde{\mathcal{N}}_{k}^{-}\right)=\tilde{\mathcal{N}}_{k}^{+}$.

Proof. Recall that $(\mathbb{C} K+\mathbb{C} D)^{\perp}=\mathfrak{h}^{\epsilon}$ and that for $j \neq 0$ an element of $\tilde{\mathcal{H}}_{j}$ (resp., $\tilde{\mathcal{H}}_{2 j}$ ) takes form $h t^{j}, h \in \mathfrak{h}$ for $\hat{\mathfrak{g}} \neq A(2 k, 2 l)^{(4)}$ (resp., for $\hat{\mathfrak{g}}=A(2 k, 2 l)^{(4)}$ ). Hence $\hat{\phi}$ satisfies (i), (ii) and the condition $\left.\hat{\phi}\right|_{\tilde{\mathcal{H}}_{2 j}}=-$ id for $\hat{\mathfrak{g}}=A(2 k, 2 l)^{(4)}$. The property (iii) follows from (i). For $\mathfrak{g}=A(2 k, 2 l)^{(4)}$ the remaining condition $\left.\hat{\phi}\right|_{\tilde{\mathcal{H}}_{4 k \pm 1}}= \pm \sqrt{-1}$ id easily follows from the fact that the spaces $\tilde{\mathcal{H}}_{4 k \pm 1}$ are one-dimensional. 
2.6.2. Lemma. There exists a commuting pair $\epsilon, \phi \in \operatorname{Aut}(\mathfrak{g})$ such that $\hat{\mathfrak{g}}=\left(\hat{\mathfrak{g}}^{\prime}\right)^{\epsilon}$ in a sense of 2.3 .3 and $\left.\phi\right|_{\mathfrak{h}}=-\mathrm{id}$.

Proof. Let $\alpha_{1}, \ldots, \alpha_{s}$ be the set of simple roots of $\mathfrak{g}$; for each $i$ choose $e_{i} \in \mathfrak{g}_{\alpha_{i}}, f_{i} \in$ $\mathfrak{g}_{-\alpha_{i}}$ such that $\left(e_{i} \mid f_{i}\right)=1$. For any collection of non-zero scalars $\left\{c_{i}\right\}_{i \in I}$ define a map $\phi(C): \mathfrak{g} \rightarrow \mathfrak{g}$ by $\phi\left(e_{i}\right)=c_{i} f_{i}, \phi\left(f_{i}\right)=(-1)^{p\left(e_{i}\right)} c_{i}^{-1} e_{i}$ and $\phi(h)=-h$ for $h \in \mathfrak{h}$. Then $\phi(C) \in \operatorname{Aut}(\mathfrak{g})$.

Let $\mathfrak{g}$ be a simple finite-dimensional Lie algebra. Then we can choose $\epsilon$ induced by an automorphism of the Dynkin diagram, that is $\epsilon\left(e_{i}\right)=e_{\sigma(i)} ; \epsilon\left(f_{i}\right)=f_{\sigma(i)}$, where $\sigma$ is an automorphism of the Dynkin diagram of $\mathfrak{g}$. Take $\phi$ which acts by - id on $\mathfrak{h}$ and interchanges $e_{i}$ with $f_{i}$. Clearly, $\phi$ commutes with $\epsilon$.

Let $\sigma$ be an automorphism of the Dynkin diagram of a finite-dimensional Kac-Moody superalgebra. Let $D=\left\{d_{i}\right\}$ be the collection of elements in $\mathbb{C}^{*}$. Denote by $\sigma(D)$ the automorphism of $\mathfrak{g}$ given by the formulas $\sigma(D)\left(e_{i}\right)=d_{i} e_{\sigma(i)}, \sigma(D)\left(f_{i}\right)=d_{i}^{-1} f_{\sigma(i)}$. Suppose that $\sigma(D)^{2}=\mathrm{id}$. It is easy to check that $\sigma(D)$ commutes with $\phi(C)$ for the following collection $C: c_{i}=d_{i}$ if $i \leq \sigma(i), c_{i}=d_{\sigma(i)}^{-1}$ if $i>\sigma(i)$.

Hence any involution of the form $\sigma(D)$ commutes with $\phi(C)$ for a suitable $C$.

Consider the case when $\hat{\mathfrak{g}}$ is a Lie superalgebra and $\hat{\mathfrak{g}} \neq A(2 k, 2 l)^{(4)}$. By $\mathrm{vdL}$, Table 4 , in this case $\epsilon^{2}=$ id and $\epsilon$ is of the form $\sigma(D)$ ( $\sigma$ is identity for $G(3)$ and is an involution in other cases). By above, $\phi(C)$ commutes with $\epsilon$ for a suitable collection $C$.

Consider the remaining case $\hat{\mathfrak{g}}=A(2 k, 2 l)^{(4)}$. By $[\underline{\mathrm{S}}]$ we can choose $\epsilon$ to be $x \mapsto-x^{s t}$, where st stands for the super-transposition in the matrix Lie superalgebra $\mathfrak{g}=A(2 k, 2 l)$. Then the restriction of $\epsilon$ to $\mathfrak{g}_{0}$ is $x \mapsto-x^{t}$, where $t$ stands for the transposition. Hence the restriction of $\epsilon$ to $\mathfrak{g}_{\overline{0}}$ is an involution.

Recall that $\epsilon$ leaves invariant a regular element of $\mathfrak{h}$ and fix the triangular decomposition $\mathfrak{g}_{\overline{0}}=\mathfrak{n}_{\overline{0},-} \oplus \mathfrak{h} \oplus \mathfrak{n}_{\overline{0}}$ defined by this regular element. Then $\epsilon$ stabilizes $\mathfrak{h}$ and $\Delta_{\overline{0}}^{+}$. Fix a Dynkin diagram for $\mathfrak{g}$ containing a unique odd node (for $i=2 k+1$ ) and a triangular decomposition which corresponds to this Dynkin diagram and is compatible with the above triangular decomposition of $\mathfrak{g}_{0}$. Choose $\left\{e_{i}, f_{i}\right\}_{i \in I}$ as above (for this triangular decomposition).

Recall that $\mathfrak{g}_{\overline{0}}=\mathfrak{s l}(2 k+1) \times \mathfrak{s l}(2 l+1) \times \mathbb{C}$. Note that $\left\{e_{i}, f_{i}\right\}_{i \in I, i \neq 2 k+1}$ generate $\mathfrak{s l}(2 k+1) \times \mathfrak{s l}(2 l+1)$. By above, the restriction of $\epsilon$ to $\mathfrak{s l}(2 k+1) \times \mathfrak{s l}(2 l+1)$ is an involution which stabilizes $\mathfrak{h}$ and $\Delta_{\overline{0}}^{+}$. Therefore this restriction takes form $\sigma(D)$, where $\sigma$ is an involution of the Dynkin diagram $A(2 k) \cup A(2 l)$. By above, for a suitable collection $C^{\prime}=\left\{c_{i}\right\}_{i \neq 2 k+1}$ the automorphism $\phi\left(C^{\prime}\right) \in \operatorname{Aut}(\mathfrak{s l}(2 k+1) \times \mathfrak{s l}(2 l+1))$ commutes with the restriction $\epsilon$ to $\mathfrak{s l}(2 k+1) \times \mathfrak{s l}(2 l+1)$.

For $\psi, \varphi \in \operatorname{Aut}(\mathfrak{g})$ set

$$
[\psi, \varphi]:=\psi \circ \varphi \circ \psi^{-1} \circ \varphi^{-1}
$$


Take any $c_{2 k+1} \in \mathbb{C}^{*}$ and let $\phi(C) \in \operatorname{Aut}(\mathfrak{g})$ be the corresponding extension of $\phi\left(C^{\prime}\right)$. Then the restrictions of $\phi(C)$ and of $\epsilon$ to $\mathfrak{s l}(2 k+1) \times \mathfrak{s l}(2 l+1)$ commute. Moreover, since $\epsilon$ stabilizes $\mathfrak{h}$ and $\left.\phi(C)\right|_{\mathfrak{h}}=-\mathrm{id}$, the restrictions of $\phi(C)$ and of $\epsilon$ to $\mathfrak{h}$ also commute. One has $\mathfrak{g}_{\overline{0}}=\mathfrak{s l}(2 k+1) \times \mathfrak{s l}(2 l+1)+\mathfrak{h}$ so $\left.[\phi(C), \epsilon]\right|_{\mathfrak{g}_{\overline{0}}}=$ id.

Recall that $\mathfrak{g}_{\overline{1}}=W \oplus W^{\prime}$, where $W, W^{\prime}$ are irreducible non-isomorphic $\mathfrak{g}_{0}$-modules. Any automorphism of $\mathfrak{g}$ which acts by id on $\mathfrak{g}_{0}$ is equal to $\psi(b)$ for $b \in \mathbb{C}^{*}$, where

$$
\left.\psi(b)\right|_{\mathfrak{g}_{\overline{0}}}=\mathrm{id},\left.\psi(b)\right|_{W}=b \mathrm{id},\left.\psi(b)\right|_{W^{\prime}}=b^{-1} \mathrm{id} .
$$

By above, $[\phi(C), \epsilon]=\psi(a)$ for some $a$. Then for any $b \in \mathbb{C}^{*}$ one has

$$
[\psi(b) \circ \phi(C), \epsilon]=\psi(b) \circ \psi(a) \circ \psi(b)^{-1} \circ[\psi(b), \epsilon]=\psi(a) \circ[\psi(b), \epsilon]
$$

Clearly, $[\psi(b), \epsilon]$ acts by id on $\mathfrak{g}_{0}$. Moreover, $\epsilon$ interchanges $W$ with $W^{\prime}$ so $[\psi(b), \epsilon]=\psi\left(b^{2}\right)$. Now taking $b$ such that $a b^{2}=1$ we get $[\psi(b) \circ \phi(C), \epsilon]=\psi(1)=$ id that is $\phi:=\psi(b) \circ \phi(C)$ commutes with $\epsilon$. Since $\phi$ acts by - id on $\mathfrak{h}$ it satisfies the required conditions.

2.7. Lemma. For any $x \in \tilde{\mathcal{H}}_{m}, y \in \tilde{\mathcal{H}}_{-m}$ one has

$$
[x, y]=m(x \mid y) K .
$$

Proof. Clearly, $[x, y]$ has zero weight so $[x, y] \in \hat{\mathfrak{h}}$. The invariance of $(-\mid-)$ gives $([x, y] \mid z)=$ $(x \mid[y, z])$ so $([x, y] \mid h)=0$ if $h \in \hat{\mathfrak{h}} \cap[\hat{\mathfrak{g}}, \hat{\mathfrak{g}}]$ and $([x, y] \mid D)=m(x \mid y)$.

2.7.1. One has decompositions

$$
\mathfrak{n}_{-}=\mathcal{N}_{-}^{-} \oplus \mathcal{H}_{-} \oplus \mathcal{N}_{-}^{+}, \mathfrak{n}=\mathcal{N}^{-} \oplus \mathcal{H} \oplus \mathcal{N}^{+},
$$

where

$$
\mathcal{H}_{-}:=\mathfrak{n}_{-} \cap \tilde{\mathcal{H}}, \quad \mathcal{N}_{-}^{ \pm}:=\mathfrak{n}_{-} \cap \tilde{\mathcal{N}}^{ \pm}, \quad \mathcal{H}:=\mathfrak{n}_{-} \cap \tilde{\mathcal{H}}, \quad \mathcal{N}^{ \pm}:=\mathfrak{n} \cap \tilde{\mathcal{N}}^{ \pm}
$$

If $\hat{\mathfrak{g}}$ is not of the type $A(2 k, 2 l)^{(4)}, \mathcal{H}_{-}$is a (even) commutative countably dimensional Lie algebra.

2.8. Verma modules. Set $\hat{\mathfrak{b}}:=\hat{\mathfrak{h}}+\hat{\mathfrak{n}}$. For $\lambda \in \hat{\mathfrak{h}}^{*}$ let $M(\lambda)$ be the Verma module of the highest weight $\lambda$ and let $v_{\lambda}$ be the canonical generator of $M(\lambda)$. A Verma module $M(\lambda)$ has a unique maximal proper submodule $M^{\prime}(\lambda)$; denote by $L(\lambda)$ the simple quotient $L(\lambda):=M(\lambda) / M^{\prime}(\lambda)$.

2.8.1. The invariant form $(-\mid-)$ gives rise to a quadratic Casimir element which acts on $M(\lambda)$ by the scalar $(\lambda, \lambda+2 \hat{\rho})$. As a result, if $L(\lambda-\nu)$ is a subquotient of $M(\lambda)$ then $2(\lambda+\hat{\rho}, \nu)=(\nu, \nu)$. 
2.8.2. Verma modules do not admit Jordan-Hölder series, since some Verma modules have an infinite length. However, so-called local series introduced in [DGK] are nice substitution for Jordan-Hölder ones. A series of weight modules $N=N_{0} \supset N_{1} \supset \ldots \supset$ $N_{m}=0$ is called local at $\nu \in \hat{\mathfrak{h}}^{*}$ if either $N_{i} / N_{i+1} \cong L\left(\lambda_{i}\right)$ for some $\lambda_{i} \geq \nu$ or $\left(N_{i} / N_{i+1}\right)_{\mu}=$ 0 for all $\mu \geq \nu$. This allows to define the multiplicity $[N: L(\lambda)]$ as the number of $i$ such that $N_{i} / N_{i+1} \cong L(\lambda)$ for a series local at some $\nu \leq \lambda$.

2.9. Projections $\mathrm{HC}$ and $\mathrm{HC}_{ \pm}$. We identify $\mathcal{U}(\hat{\mathfrak{h}})$ with $\mathcal{S}(\hat{\mathfrak{h}})$. The triangular decomposition $\hat{\mathfrak{g}}=\hat{\mathfrak{n}}_{-} \oplus \hat{\mathfrak{h}} \oplus \hat{\mathfrak{n}}$ gives rise to the Harish-Chandra projection $\mathrm{HC}: \mathcal{U}(\hat{\mathfrak{g}}) \rightarrow \mathcal{U}(\hat{\mathfrak{h}})=\mathcal{S}(\hat{\mathfrak{h}})$ along the kernel $\mathcal{U}(\hat{\mathfrak{g}}) \hat{\mathfrak{n}}^{+}+\hat{\mathfrak{n}}^{-} \mathcal{U}(\hat{\mathfrak{g}})$. The restriction of HC to $\mathcal{U}(\hat{\mathfrak{g}})^{\hat{\mathfrak{h}}}$ is an algebra homomorphism.

\subsubsection{Algebra $\mathcal{S}$. Set}

$$
\mathcal{S}:=\mathcal{U}\left(\mathcal{H}_{-}\right)
$$

The algebra $\mathcal{S}$ inherits the grading $\mathcal{S}=\oplus_{s \geq 0} \mathcal{S}_{-s \delta}$.

Let $\hat{\mathfrak{g}} \neq A(2 k, 2 l)^{(4)}$. Then $\mathcal{H}_{-}$is pure even and commutative so $\mathcal{S}$ is the symmetric algebra of $\mathcal{H}_{-}$. In this case $\mathcal{S}$ has a natural grading of symmetric algebra $\mathcal{S}=\oplus \mathcal{S}^{k}$, where $\mathcal{S}^{k}$ is spanned by the monomials of degree $k$. We set $\mathcal{S}^{\geq k}:=\sum_{j=k}^{\infty} \mathcal{S}^{j}$.

2.9.2. Projection $\mathrm{HC}_{ \pm}$. The decomposition $\hat{\mathfrak{n}}_{-}=\mathcal{N}_{-}^{+} \oplus \mathcal{H}_{-} \oplus \mathcal{N}_{-}^{-}$gives rise to the projections $\mathrm{HC}_{ \pm}: \mathcal{U}\left(\hat{\mathfrak{n}}_{-}\right) \rightarrow \mathcal{S}$, where

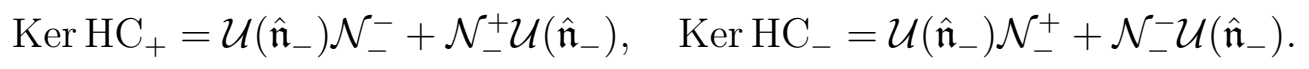

Recall that $\mathfrak{h}^{\prime}=\hat{\mathfrak{h}} \cap[\hat{\mathfrak{g}}, \hat{\mathfrak{g}}]$ and $\mathcal{H}_{-}=\hat{\mathfrak{n}}_{-}^{\mathfrak{h}^{\prime}}$. Observe that $\left(\text { Ker HC} \mathrm{HC}_{+}\right)^{\mathfrak{h}^{\prime}}=\mathcal{U}\left(\hat{\mathfrak{n}}_{-}\right) \mathcal{N}_{-}^{-}=$ $\mathcal{N}_{-}^{+} \mathcal{U}\left(\hat{\mathfrak{n}}_{-}\right)$and similarly $\left(\operatorname{Ker} \mathrm{HC}_{+}\right)^{\mathfrak{h}^{\prime}}=\mathcal{U}\left(\hat{\mathfrak{n}}_{-}\right) \mathcal{N}_{-}^{+}=\mathcal{N}_{-}^{-} \mathcal{U}\left(\hat{\mathfrak{n}}_{-}\right)$. Thus the restriction of $\mathrm{HC}_{ \pm}$to $\mathcal{U}\left(\hat{\mathfrak{n}}_{-}\right)^{\mathfrak{h}^{\prime}}\left(\mathfrak{h}^{\prime}=\hat{\mathfrak{h}} \cap[\hat{\mathfrak{g}}, \hat{\mathfrak{g}}]\right)$ are algebra homomorphism.

A Verma module $M(\lambda)$ can be canonically identified with $\mathcal{U}\left(\hat{\mathfrak{n}}_{-}\right)$. We denote by $\mathrm{HC}_{ \pm}$: $M(\lambda) \rightarrow \mathcal{S}$ the corresponding maps obtained via this identification. Set

$$
\breve{M}(\lambda):=\mathcal{U}\left(\hat{\mathfrak{n}}_{-}\right)^{\mathfrak{h}^{\prime}} v_{\lambda}
$$

and note that $\breve{M}(\lambda)=\sum_{s} M(\lambda)_{\lambda-s \delta}$. For the maps $\mathrm{HC}_{ \pm}: M(\lambda) \rightarrow \mathcal{S}$ the above observation gives

$$
\left(\mathrm{Ker} \mathrm{HC}_{+} \cap \breve{M}(\lambda)\right) \subset \mathcal{N}_{-}^{+} M(\lambda), \quad(\text { Ker HC}-\cap \breve{M}(\lambda)) \subset \mathcal{N}_{-}^{-} M(\lambda) .
$$

2.10. Shapovalov form. Let us recall the standard construction of the Shapovalov form for $\hat{\mathfrak{g}}$. 
2.10.1. Choice of antiautomorphism. Call a linear endomorphism $\sigma$ of a superalgebra a "naive" antiautomorphism if $\sigma$ is invertible and $\sigma([x y])=[\sigma(y), \sigma(x)]$. By construction, any Kac-Moody superalgebra admits a "naive" anti-involution $\sigma$ which preserves the elements of a Cartan subalgebra ( $\sigma$ acts on Cartan generators by $\sigma\left(e_{i}\right)=f_{i}$ ). One has

$$
\sigma^{2}=\mathrm{id},\left.\sigma\right|_{\hat{\mathfrak{h}}}=\mathrm{id}, \sigma(\hat{\mathfrak{n}})=\hat{\mathfrak{n}}_{-},(\sigma(b) \mid \sigma(a))=(a \mid b)
$$

All the above properties except the last one follows from the definition of $\sigma$. To verify the last formula note that $(a \mid b)^{\prime \prime}:=(\sigma(b) \mid \sigma(a))$ is an even invariant bilinear form and both $(-\mid-),(-\mid-)^{\prime \prime}$ induce maps $\varphi, \varphi^{\prime \prime}: \hat{\mathfrak{g}} \rightarrow \hat{\mathfrak{g}}^{*}$. The kernel of $\varphi-\varphi^{\prime \prime}$ is an ideal containing $\hat{\mathfrak{h}}$ since $\left.\sigma\right|_{\hat{\mathfrak{h}}}=\mathrm{id}$. Hence $\varphi=\varphi^{\prime \prime}$ and thus $(\sigma(b) \mid \sigma(a))=(a \mid b)$ as required.

2.10.2. Define a form $S: \mathcal{U}\left(\hat{\mathfrak{n}}_{-}\right) \otimes \mathcal{U}\left(\hat{\mathfrak{n}}_{-}\right) \rightarrow \mathcal{S}(\hat{\mathfrak{h}})$ by setting $S(x, y):=\operatorname{HC}(\sigma(x) y)$. Using the natural identification of a Verma module $M(\lambda)$ with $\mathcal{U}\left(\hat{\mathfrak{n}}_{-}\right)$, one easily sees that the maximal proper submodule $M^{\prime}(\lambda)$ of $M(\lambda)$ coincides with the kernel of the evaluated form $S(\lambda): \mathcal{U}\left(\hat{\mathfrak{n}}_{-}\right) \otimes \mathcal{U}\left(\hat{\mathfrak{n}}_{-}\right) \rightarrow \mathbb{C}$.

Notice that $S(x, y)=0$ if $x$ and $y$ have distinct weights. Thus $S=\sum_{\nu \in \hat{Q}^{+}} S_{\nu}$ where $S_{\nu}$ is the restriction of $S$ to $\mathcal{U}\left(\mathfrak{n}_{-}\right)_{-\nu} \otimes \mathcal{U}\left(\mathfrak{n}_{-}\right)_{-\nu}$. By the above, $\operatorname{dim} L(\lambda)_{\lambda-\nu}=\operatorname{codim} \operatorname{ker} S_{\nu}(\lambda)$.

2.10.3. The determinant of $S_{\nu}$ is defined up to a non-zero scalar factor and is computed in [KK], GK]:

$$
\operatorname{det} S_{\nu}(\lambda)=\prod_{m=1}^{\infty} \prod_{\gamma \in \hat{\Delta}^{+}} \phi_{m \gamma}^{(-1)^{(m-1) p(\gamma)} \tau(\nu-m \gamma)}(\lambda),
$$

where $\hat{\Delta}^{+}$stands for the multiset of positive roots, $\phi_{m \gamma}(\lambda)=2(\lambda+\hat{\rho}, m \gamma)-(m \gamma, m \gamma)$, and $\tau: \hat{Q}^{+} \rightarrow \mathbb{Z}_{\geq 0}$ stands for the Kostant partition function given by $\tau(\nu)=\operatorname{dim} \mathcal{U}(\hat{\mathfrak{n}})_{\nu}=$ $\operatorname{dim} M(\lambda)_{\lambda-\nu}$.

Note that for any $\gamma \in \hat{\Delta}_{i m}, m \geq 1$ one has $\phi_{m \gamma}(\lambda)=0$ iff $(\lambda+\hat{\rho}, \delta)=0$.

2.10.4. Remark. Note that $\sigma\left(\mathcal{N}^{+}\right)=\mathcal{N}_{-}^{-}, \sigma\left(\mathcal{N}^{-}\right)=\mathcal{N}_{-}^{+}$because $\left.\sigma\right|_{\hat{\mathfrak{h}}}=$ id. From (3) it follows that $\breve{M}(\lambda)^{\mathcal{N}^{ \pm}}$is orthogonal to Ker $\mathrm{HC}_{\mp}$ with respect to the Shapovalov form:

$$
S(\lambda)\left(\breve{M}(\lambda)^{\mathcal{N}^{+}}, \text {Ker HC}-\right)=S(\lambda)\left(\breve{M}(\lambda)^{\mathcal{N}^{-}}, \text {Ker HC}+\right)=0
$$

2.11. Characters. For a diagonalizable $\hat{\mathfrak{h}}$-module $M$ we denote by $\Omega(M)$ the set of weights of $M$ and by $M_{\mu}$ the weight space of weight $\mu$. We say that a module $M$ admits a character $\operatorname{ch} M=\sum_{\mu} \operatorname{dim} M_{\mu} e^{\mu}$ if $M$ is a diagonalizable $\hat{\mathfrak{h}}$-module with the finite dimensional weight spaces.

If $v \in M$ is a weight vector we denote its weight by wt $v$; for $u \in \mathcal{U}(\mathfrak{g})$ we denote by wt $u$ its weight with respect to the adjoint action of $\hat{\mathfrak{h}}$. 
2.11.1. For a finite set $X:=\left\{\lambda_{i}\right\}_{i=1}^{r} \subset \hat{\mathfrak{h}}$ let $C_{X}$ be the collection of elements of the form $\sum_{i=1}^{r} \sum_{\mu<\lambda_{i}} c_{\mu} e^{\mu}$, where $c_{\mu} \in \mathbb{Z}, c_{\mu} \geq 0$. Let $C$ be the union of $C_{X}$. Note that $x, y \in C$ implies $x+y, x y \in C$ and introduce the partial order on $C$ by $x \geq y$ if $x-y \in C$. In all our examples ch $M$ belongs to $C$.

2.11.2. Set $M_{>\mu}:=\sum_{\nu>\mu} M_{\nu}$ and define $M_{\geq \mu}$ similarly. For any map $f: M \rightarrow N$ we denote by $f_{\mu}$ (resp., $f_{>\mu}, f_{\geq \mu}$ ) the restriction of $f$ to $M_{\mu}$ (resp., $M_{>\mu}, M_{\geq \mu}$ ).

2.11.3. Recall that $\hat{\mathfrak{g}}=[\hat{\mathfrak{g}}, \hat{\mathfrak{g}}] \oplus \mathbb{C} D$. As $[\hat{\mathfrak{g}}, \hat{\mathfrak{g}}]$-modules for any $s$ one has $M(\lambda) \cong M(\lambda-s \delta)$ and so $M^{\prime}(\lambda) \cong M^{\prime}(\lambda-s \delta)$. As a consequence, $\operatorname{ch} L(\lambda-s \delta)=e^{-s \delta} \operatorname{ch} L(\lambda)$.

\subsection{Generic critical weights. Set}

$$
\Lambda:=\{\lambda \in \hat{\mathfrak{h}} \mid[M(\lambda): L(\lambda-\nu)]=0 \text { for } \nu \notin \mathbb{N} \delta\}, \quad \Lambda_{\text {crit }}=\{\lambda \in \Lambda \mid(\lambda+\hat{\rho}, \delta)=0\} .
$$

From the formula for Shapovalov determinants (see 2.10.3) we see that $\lambda \in \Lambda \backslash \Lambda_{\text {crit }}$ iff $M(\lambda)$ is simple. For $\lambda \in \Lambda_{\text {crit }}$ the Verma module $M(\lambda)$ is not irreducible and all its subquotients are of the form $L(\lambda-s \delta)$ for $s \geq 0$.

By [KK], Thm. $2 \lambda \in \Lambda_{\text {crit }}$ iff $(\lambda+\hat{\rho}, \delta)=0$ and $\lambda$ is not a root of other factors of Shapovalov determinants, i.e $\phi_{m \gamma}(\lambda) \neq 0$ for $\gamma \notin \Delta_{i m}$.

Call $\lambda \in \Lambda_{\text {crit }}$ a generic critical weight if $\lambda \in \Lambda_{\text {crit }}$.

\subsubsection{We will use the following lemma.}

Lemma. Take $\lambda \in \Lambda$. If $v^{\prime} \in M(\lambda)_{\lambda-s \delta}$ is such that $\left(\mathcal{N}^{+}+\mathcal{H}\right) v^{\prime}=0$ or $\left(\mathcal{N}^{-}+\mathcal{H}\right) v^{\prime}=$ 0 then $v^{\prime}$ is singular.

Proof. Assume that $\left(\mathcal{N}^{+}+\mathcal{H}\right) v^{\prime}=0$. Since $\hat{\mathfrak{n}}=\left(\mathcal{N}^{+}+\mathcal{H}\right) \oplus \mathcal{N}^{-}$one has $\mathcal{U}(\hat{\mathfrak{n}}) v^{\prime}=\mathcal{U}\left(\mathcal{N}^{-}\right) v^{\prime}$. The weight vectors in $\mathcal{U}\left(\mathcal{N}^{-}\right) v^{\prime}$ which are not proportional to $v^{\prime}$ have weights of the form $\lambda-\mu$ where $\mu \notin \mathbb{N} \delta$; these vectors are not singular, since $\lambda \in \Lambda$. Since $\mathcal{U}(\hat{\mathfrak{n}}) v^{\prime}$ contains at least one singular weight vector, $v^{\prime}$ is singular.

The proof for $\left(\mathcal{N}^{-}+\mathcal{H}\right) v^{\prime}=0$ is similar.

2.12.2. Remark. Recall that each factor of a Shapovalov determinant corresponds to a hyperplane $\gamma_{k \alpha}$ in $\hat{\mathfrak{h}}^{*}$; the set of generic critical weights consists of the points of the hyperplane $(\lambda+\hat{\rho}, \delta)=0$ which do not lie on other hyperplanes $\gamma_{k \alpha}$. From the formula given in 2.10 .3 one sees that $\lambda$ is a generic critical weight iff

$$
(\lambda+\hat{\rho}, \delta)=0 \&(\lambda+\hat{\rho}, \alpha) \neq k(\alpha, \alpha) \text {, where } \alpha \in \hat{\Delta}^{+}, k \in \mathbb{Z}_{>0} \& k \text { is odd if } \alpha \text { is odd. }
$$




\section{Towards the Proof of Theorems 1.1.1,1.1.3}

In this section we reduce Theorems 1.1.1]1.1.3 to the assertions $3.2(\mathrm{~A}),(\mathrm{B})$.

3.1. Algebra structure on $\breve{M}(\lambda)^{\hat{\mathfrak{n}}}$. Recall that $\breve{M}(\lambda)=\sum_{s} M(\lambda-s \delta)$. Identify $\operatorname{End}_{[\hat{\mathfrak{g}}, \hat{\mathfrak{g}}]}(M(\lambda))$ with $\breve{M}(\lambda)^{\hat{\mathfrak{n}}}$ via the map $\phi \mapsto \phi\left(v_{\lambda}\right)$ and endow $\breve{M}(\lambda)^{\hat{\mathfrak{n}}}$ with the algebra structure via this bijection.

\subsubsection{Lemma. The restrictions $\mathrm{HC}_{ \pm}: \breve{M}(\lambda)^{\hat{\mathfrak{n}}} \rightarrow \mathcal{S}$ are algebra homomorphisms.}

Proof. Let $\iota$ stands for the natural identification $M(\lambda)$ with $\mathcal{U}\left(\hat{\mathfrak{n}}_{-}\right)$. Clearly, the algebra structure on $\breve{M}(\lambda)^{\hat{\mathfrak{n}}}$ is compatible with $\iota$, i.e. $\iota(x) \iota(y)=\iota(x y)$.

Notice that $\iota$ maps $\breve{M}(\lambda)$ to $\mathcal{U}\left(\hat{\mathfrak{n}}_{-}\right)^{\mathfrak{h}^{\prime}}$, where $\mathfrak{h}^{\prime}=\hat{\mathfrak{h}} \cap[\hat{\mathfrak{g}}, \hat{\mathfrak{g}}]$. Now the assertion follows from the fact that the restrictions of $\mathrm{HC}_{ \pm}$to $\mathcal{U}\left(\hat{\mathfrak{n}}_{-}\right)^{\mathfrak{h}^{\prime}}$ is an algebra homomorphism.

3.2. Claim. Take $\hat{\mathfrak{g}} \neq A(2 k, 2 l)^{(4)}$ and $\lambda \in \Lambda_{\text {crit }}$. The following assertions imply Theorems 1.1.1, 1.1.3 and the formula (1):

(A) The restrictions of $\mathrm{HC}_{ \pm}$to the set of singular vectors of $M(\lambda)^{k}$ contain $\mathcal{S} \geq k$.

(B) $\forall k, s \quad\left[M(\lambda)^{k}: L(\lambda-s \delta)\right]=\operatorname{dim} \mathcal{S}_{-s \delta}^{\geq k}$.

We prove this claim in 3.2 .13 .2 .5 below.

3.2.1. Theorem 1.1.1 (i) claims that $M(\lambda)$ does not have subsingular vectors, i.e.

$$
\forall \mu \in \hat{\mathfrak{h}}^{*} \quad[M(\lambda): L(\mu)]=\operatorname{dim} M(\lambda)_{\mu}^{\hat{\mathfrak{n}}}
$$

and that any submodule of $M(\lambda)$ is generated by its singular vectors. Combining $(\mathrm{A})$ and (B) for $k=0$ we obtain (5).

Let us show that (5) implies that any submodule of $M(\lambda)$ is generated by its singular vectors. Let $\mathcal{Y}$ be the set of modules in category $\mathcal{O}$ which do not have subsingular vectors: $\mathcal{Y}:=\left\{M \in \mathcal{O} \mid \forall \mu[M: L(\mu)]=\operatorname{dim} M_{\mu}^{\hat{\mathfrak{n}}}\right\}$. Take $M \in \mathcal{Y}$ and let $N$ is a submodule of $M$. For any $\mu \in \hat{\mathfrak{h}}^{*}$ one has

$$
[M: L(\mu)]=[N: L(\mu)]+[M / N: L(\mu)] \geq \operatorname{dim} N_{\mu}^{\hat{\mathfrak{n}}}+\operatorname{dim}(M / N)_{\mu}^{\hat{\mathfrak{n}}} \geq \operatorname{dim} M_{\mu}^{\hat{\mathfrak{n}}} .
$$

Therefore $N, M / N \in \mathcal{Y}$ and, moreover, $\operatorname{dim} M_{\mu}^{\hat{\mathfrak{n}}}=\operatorname{dim} N_{\mu}^{\hat{\mathfrak{n}}}+\operatorname{dim}(M / N)_{\mu}^{\hat{\mathfrak{n}}}$.

By (5), $M(\lambda) \in \mathcal{Y}$. Let $M$ be any submodule of $M(\lambda)$ and let $N$ be the submodule generated by $M^{\hat{\mathfrak{n}}}$. By above $M \in \mathcal{Y}$ and $\operatorname{dim}(M / N)_{\mu}^{\hat{\mathfrak{n}}}=0$ for any $\mu$. Hence $M / N=0$ as required. 
3.2.2. Theorem 1.1.1 (ii) asserts that $\mathrm{HC}_{ \pm}$induce bijections $\mathrm{HC}_{ \pm}: M(\lambda)^{\hat{\mathfrak{n}}} \stackrel{\sim}{\longrightarrow} \mathcal{S}$ and that these bijections are algebra isomorphisms. By (A) the maps $\mathrm{HC}_{ \pm}: M(\lambda)^{\hat{\mathfrak{n}}} \rightarrow \mathcal{S}$ are surjective. Combining (5) with (B) for $k=0$ we get $e^{-\lambda} \operatorname{ch} M(\lambda)^{\hat{\mathfrak{n}}}=\operatorname{ch} \mathcal{S}$ and this implies the injectivity. The fact that these bijections are algebra isomorphisms follows from Lemma 3.1.1.

3.2.3. Theorem 1.1.1 (iii) asserts that the submodule generated by a singular vector $v \in M(\lambda)$ is isomorphic a Verma module.

Let $\phi \in \operatorname{End}_{[\hat{\mathfrak{g}}, \hat{\mathfrak{g}}]}(M(\lambda))$ be such that $\phi\left(v_{\lambda}\right)=v$. If $\operatorname{Ker} \phi$ is non-zero, it contains a singular vector $w$. Then $0=\mathrm{HC}_{+}(\phi(w))=\mathrm{HC}_{+}(w) \mathrm{HC}_{+}(v)$ by Lemma 3.1.1, By 3.2.2, $\mathrm{HC}_{+}(w), \mathrm{HC}_{+}(v)$ are non-zero. Since $\mathcal{S}$ is a polynomial algebra this gives a contradiction. Hence $\operatorname{Ker} \phi=0$ and this implies (iii).

3.2.4. Let us deduce the formula (1): $\operatorname{ch} N=\operatorname{ch} L(\lambda) \cdot \operatorname{ch~} \operatorname{HC}_{+}\left(N^{\hat{\mathfrak{n}}}\right)$, where $N$ is a submodule of $M(\lambda)$.

By 3.2.1, $[N: L(\mu)]=\operatorname{dim} N_{\mu}^{\hat{\mathfrak{n}}}$. Since the weights of singular vector are of the form $\lambda-s \delta$, one has $\operatorname{ch} N=\operatorname{ch} L(\lambda) \cdot\left(e^{-\lambda} \operatorname{ch} N^{\hat{\mathfrak{n}}}\right)$. By 3.2.2, $e^{-\lambda} \operatorname{ch} N^{\hat{\mathfrak{n}}}=\operatorname{ch~} \mathrm{HC}_{+}\left(N^{\hat{\mathfrak{n}}}\right)$ and this gives the required formula.

3.2.5. Recall that Theorem 1.1.3 means that $\mathrm{HC}_{ \pm}$induce bijections $\mathrm{HC}_{ \pm}:\left(M(\lambda)^{k}\right)^{\hat{\mathfrak{n}}} \stackrel{\sim}{\longrightarrow}$ $\mathcal{S} \geq k$. By 3.2.2, the restrictions of $\mathrm{HC}_{ \pm}$to $\left(M(\lambda)^{k}\right)^{\hat{\mathfrak{n}}}$ are injective; by (A) the images contain $\mathcal{S}^{\geq k}$. Finally, combining (5) with (B) we get $e^{-\lambda} \operatorname{ch}\left(M(\lambda)^{k}\right)^{\hat{\mathfrak{n}}}=\operatorname{ch} \mathcal{S}^{\geq k}$. Hence the images coincide with $\mathcal{S}^{\geq k}$ as required.

\subsection{Corollary. For $\lambda$ at the critical level one has $\operatorname{ch} L(\lambda) \operatorname{ch} \mathcal{S} \leq \operatorname{ch} M(\lambda)$.}

Proof. By (A) for $\lambda \in \Lambda_{\text {crit }}$ one has $e^{-\lambda} \operatorname{ch} M(\lambda)^{\hat{\mathfrak{n}}} \geq \operatorname{ch} \mathcal{S}$. Since $\Lambda_{\text {crit }}$ is Zariski dense at the critical level, $\operatorname{dim} M(\lambda)_{\lambda-s \delta}^{\hat{\mathfrak{n}}} \geq \operatorname{dim} \mathcal{S}_{s \delta}$ for any $\lambda$ at the critical level. In the notation of 2.8.2 we have

$$
\begin{aligned}
\operatorname{ch} M(\lambda) & =\sum_{\nu \in \hat{Q}^{+}}[M(\lambda): L(\lambda-\nu)] \operatorname{ch} L(\lambda-\nu) \geq \sum_{s=0}^{\infty}[M(\lambda): L(\lambda-s \delta)] \operatorname{ch} L(\lambda-s \delta) \\
& ={ }^{\text {by } 2.11 .3} \sum_{s=0}^{\infty}[M(\lambda): L(\lambda-s \delta)] e^{-s \delta} \operatorname{ch} L(\lambda) \geq \operatorname{ch} L(\lambda) \sum_{s=0}^{\infty} \operatorname{dim} \mathcal{S}_{-s \delta} e^{-s \delta} \\
& =\operatorname{ch} L(\lambda) \operatorname{ch} \mathcal{S} .
\end{aligned}
$$




\section{JANTZEN FILTRATION}

In this section we recall the construction of the Jantzen filtration (see [Ja]) and then describe this filtration for a vacuum module over a Heisenberg algebra.

4.1. Notation. Let $A$ be the localization of $\mathbb{C}[x]$ by the ideal generated by $x: A:=$ $\mathbb{C}[x]_{(x)}$. For a Lie algebra $\mathfrak{p}$ and a $\mathfrak{p}$-module $N$ set $\mathfrak{p}_{A}:=\mathfrak{p} \otimes_{\mathbb{C}} A, N_{A}:=N \otimes_{\mathbb{C}} A$.

4.1.1. Choice of $\xi$. Choose an element $\xi \in \hat{\mathfrak{h}}^{*}$ such that for any $\nu \in \hat{Q}^{+}$the Shapovalov determinant $\operatorname{det} S_{\nu}(\lambda+t \xi)$, viewed as a polynomial in $t$, is not identically equal to zero.

4.1.2. Introduce a Verma module $M(\lambda+x \xi)$ over $\hat{\mathfrak{g}}_{A}$ as follows.

Define the action of $(\mathfrak{n}+\mathfrak{h})$ on $A: \mathfrak{n}$ acts trivially and $h \in \mathfrak{h}$ acts by the multiplication to $(\lambda+x \xi)(h)=\lambda(h)+x \xi(h)$. Now $M(\lambda+x \xi)$ is the following $\hat{\mathfrak{g}}_{A}$ module

$$
M(\lambda+x \xi):=\operatorname{Ind}_{\mathfrak{n}+\mathfrak{h}}^{\mathfrak{g}} A .
$$

Set

$$
\breve{M}(\lambda+x \xi)=\sum_{s=0}^{\infty} M(\lambda+x \xi)_{\lambda+x \xi-s \delta} .
$$

4.2. Definition of Jantzen filtration. The Shapovalov form $S: \mathcal{U}\left(\hat{\mathfrak{n}}_{-}\right) \otimes \mathcal{U}\left(\hat{\mathfrak{n}}_{-}\right) \rightarrow$ $\mathcal{S}(\hat{\mathfrak{h}})$ induces the Shapovalov form $S_{A}: \mathcal{U}\left(\hat{\mathfrak{n}}_{-, A}\right) \otimes \mathcal{U}\left(\hat{\mathfrak{n}}_{-, A}\right) \rightarrow \mathcal{S}\left(\hat{\mathfrak{h}}_{A}\right)$ and the evaluated Shapovalov form $S_{A}(\lambda+x \xi): M(\lambda+x \xi) \otimes M(\lambda+x \xi) \rightarrow A$. For $k \in \mathbb{Z}_{\geq 0}$, set

$$
\mathcal{F}(M(\lambda+x \xi))^{k}:=\left\{v \in M(\lambda+x \xi) \mid S_{A}(\lambda+x \xi)\left(v, v^{\prime}\right) \in t^{k} A \quad \forall v^{\prime}\right\} .
$$

This defines a decreasing filtration. It is easy to see that each term $\mathcal{F}(M(\lambda+x \xi))^{k}$ is a submodule of $M(\lambda+x \xi)$. The weight spaces of $M(\lambda+x \xi)$ are free of finite rank $A$-modules and the determinant of the restriction of $S_{A}(\lambda+x \xi)$ to the weight space $\lambda+x \xi-\nu$ i equal (up to a non-zero factor in $A$ ) to $\operatorname{det} S_{\nu}(\lambda+x \xi)$ and this is non-zero due to the condition on $\xi$. As a result, $\cap_{k=0}^{\infty} \mathcal{F}^{k}(M(\lambda+x \xi))=0$.

Specializing this filtration at $x=0$ we obtain the Jantzen filtration $M^{k}(\lambda)$ on $M(\lambda)$. The terms $M^{k}(\lambda)$ form a decreasing filtration by submodules of $M(\lambda)$ having zero intersection. One readily sees that $M^{0}(\lambda)=M(\lambda)$ and that $M^{1}(\lambda)$ coincides with the maximal proper submodule of $M(\lambda)$.

Define $v_{x}: A \rightarrow \mathbb{N}$ by $a \in(x)^{v_{x}(a)}, a \notin(x)^{v_{x}(a)+1}$ for any $a \in A$. The Jantzen sum formula ([Ja], Lem.3) claims that

$$
\forall \mu \quad \sum_{k \geq 1} \operatorname{dim} M(\lambda)_{\lambda-\mu}^{k}=v_{x}\left(\operatorname{det} S_{A ; \mu}(\lambda+x \xi)\right) .
$$

Observe that for each $\mu$ one has $M(\lambda)_{\mu}^{k}=0$ for $k>>0$. 
4.2.1. Let $\lambda$ be a generic critical weight. For $\hat{\mathfrak{g}} \neq A(22 k, 2 l)^{(4)}$ the set $\hat{\Delta}_{i m, \overline{1}}^{+}$is empty and the Jantzen sum formula gives

$$
\sum_{k \geq 1} \operatorname{ch} M(\lambda)^{k}=\operatorname{ch} M(\lambda) \cdot \sum_{m=1}^{\infty} \sum_{\alpha \in \hat{\Delta}_{i m}^{+}} e^{-m \alpha}=\operatorname{ch} M(\lambda) \cdot \sum_{\alpha \in \hat{\Delta}_{i m}^{+}} \frac{e^{-\alpha}}{1-e^{-\alpha}} .
$$

4.3. Jantzen filtration for modules over a Heisenberg algebra. Let $\hat{\mathfrak{g}} \neq A(2 k, 2 l)^{(4)}$. In this case $\mathcal{H}_{-}+\mathbb{C} K+\mathcal{H}$ is a Heisenberg algebra. For $k \in \mathbb{C}$ let $V^{k}$ be a vacuum module over $\mathcal{H}_{-}+\mathbb{C} K+\mathcal{H}$ :

$$
V^{k}=\mathcal{U}\left(\mathcal{H}_{-}+\mathbb{C} K+\mathcal{H}\right) \otimes_{\mathcal{U}(\mathbb{C} K+\mathcal{H})} \mathbb{C} v_{k}, \quad K v_{k}=k v_{k}, \mathcal{H} v_{k}=0 .
$$

View $V^{k}$ as a graded module via the natural identification of $V^{k}$ with $\mathcal{S}=\mathcal{U}\left(\mathcal{H}_{-}\right)$; note that the homogeneous components of $V^{k}$ are finite dimensional.

Define the Shapovalov form $\mathcal{S} \otimes \mathcal{S} \rightarrow \mathbb{C}[K]$ as in 2.10.2. It is easy to see that $V^{k}$ is simple for $k \neq 0$ and that for $V^{0}$ the Jantzen filtration $\mathcal{F}^{k}\left(V^{0}\right)$ identifies with the adic filtration $\left\{\mathcal{S}^{\geq k}\right\}$ of $\mathcal{S}=\mathcal{U}\left(\mathcal{H}_{-}\right)$. Set $\operatorname{ch}_{q} \mathcal{S}=\prod_{\alpha \in \hat{\Delta}_{i m}^{+}}\left(1-q e^{-\alpha}\right)^{-1}$. Then

$$
\sum_{k \geq 1} \operatorname{ch} \mathcal{S}^{\geq k}=\left.\frac{\partial \operatorname{ch}_{q} \mathcal{S}}{\partial q}\right|_{q=1}
$$

Thus

$$
\sum_{k \geq 1} \operatorname{ch} \mathcal{F}^{k}\left(V^{0}\right)=\sum_{k \geq 1} \operatorname{ch} \mathcal{S}^{\geq k}=\operatorname{ch} \mathcal{S} \sum_{\alpha \in \hat{\Delta}_{i m}^{+}} \frac{e^{-\alpha}}{1-e^{-\alpha}}
$$

4.3.1. Let $\lambda$ be a generic critical weight. Combining (6) and (77) we get

$$
\sum_{k \geq 1} \operatorname{ch} M(\lambda)^{k}=\frac{\operatorname{ch} M(\lambda)}{\operatorname{ch} \mathcal{S}} \sum_{k \geq 1} \operatorname{ch} \mathcal{S}^{\geq k}
$$

Recall that the Kac-Kazhdan character formula states that $\operatorname{ch} L(\lambda)=\frac{\operatorname{ch} M(\lambda)}{\operatorname{ch} \mathcal{S}}$ and that Theorem 1.1.3 states that $\operatorname{ch} M(\lambda)^{k}=\operatorname{ch} L(\lambda) \operatorname{ch} \mathcal{S}^{\geq k}$ for all $k$. We will use the formula (8) in the proof of 3.2 (B).

\section{A technical Lemma}

In this section $\hat{\mathfrak{g}}$ is an affine Lie superalgebra with a symmetrizable Cartan matrix (we do not exclude the case $\left.A(2 k, 2 l)^{(4)}\right)$. We prove Lemma 5.4.2, this lemma is used in the explicit construction of a singular vector in Sect. 7 .

In this section $\mathfrak{q}, \mathfrak{q}-$ are any subalgebras of $\hat{\mathfrak{n}}$ satisfying

$$
\mathcal{N}^{+} \subseteq \mathfrak{q} \subseteq \mathcal{N}^{+}+\mathcal{H}, \quad \mathcal{N}^{-} \subseteq \mathfrak{q}^{-} \subseteq \mathcal{N}^{-}+\mathcal{H}
$$


5.1. Notation. Let $h^{\prime} \in \hat{\mathfrak{h}}$ be the defining element for the triangular decomposition $[\hat{\mathfrak{g}}, \hat{\mathfrak{g}}]=\tilde{\mathcal{N}}^{-} \oplus \tilde{\mathcal{H}} \oplus \tilde{\mathcal{N}}^{+}$(see 2.5). Recall that $\Omega\left(\tilde{\mathcal{N}}^{+}\right)=\left\{\alpha \in \hat{\Delta} \mid \operatorname{Re} \alpha\left(h^{\prime}\right)>0\right\}$. Set $\hat{Q}^{-}:=\Omega\left(\mathcal{U}\left(\hat{\mathfrak{n}}^{-}\right)\right)=-\hat{Q}^{+}$and

$$
\hat{Q}_{+}^{-}:=\left\{\nu \in \hat{Q}^{-} \mid \operatorname{Re} \nu\left(h^{\prime}\right)>0\right\}, \quad \hat{Q}_{-}^{-}:=\left\{\nu \in \hat{Q}^{-} \mid \operatorname{Re} \nu\left(h^{\prime}\right)<0\right\} .
$$

Retain notation of 2.12 .

\subsection{Lemma. For any $\lambda \in \Lambda$ one has}

$$
\begin{aligned}
& H^{m}(\mathfrak{q}, L(\lambda))_{\lambda+\mu}=0 \text { and } H^{m}(\mathfrak{q}, M(\lambda))_{\lambda+\mu}=0 \text { for } m=0,1 \text { and } \mu \in \hat{Q}_{-}^{-}, \\
& H^{m}\left(\mathfrak{q}^{-}, L(\lambda)\right)_{\lambda+\mu}=0 \text { and } H^{m}\left(\mathfrak{q}^{-}, M(\lambda)\right)_{\lambda+\mu}=0 \text { for } m=0,1 \text { and } \mu \in \hat{Q}_{+}^{-} .
\end{aligned}
$$

Proof. Recall that $\sigma\left(\mathcal{N}^{+}\right)=\mathcal{N}_{-}^{-}$. Lemma 9.1 reduces the first formula $H^{m}(\mathfrak{q}, L(\lambda))_{\lambda+\mu}=$ 0 to the inclusions

$$
L(\lambda)_{\lambda+\mu} \subset \mathcal{N}_{-}^{-} L(\lambda), \quad M^{\prime}(\lambda)_{\lambda+\mu} \subset \mathcal{N}_{-}^{-} M^{\prime}(\lambda),
$$

where $M^{\prime}(\lambda)$ is the maximal proper submodule of $M(\lambda)$.

Recall that $\operatorname{Re}\left((s \delta+\mu) h^{\prime}\right)<0$. Observe that if $\nu$ is a weight of $\mathcal{U}\left(\mathcal{N}_{-}^{+}+\mathcal{H}_{-}\right)$then $\operatorname{Re} \nu\left(h^{\prime}\right) \geq 0$; thus $\nu$ is not of the form $s \delta+\mu$ for $s \in \mathbb{Z}$ :

$$
\Omega\left(\mathcal{U}\left(\mathcal{N}_{-}^{+}+\mathcal{H}_{-}\right)\right) \cap(\mu+\mathbb{Z} \delta)=\emptyset .
$$

Both inclusions can be easily deduced from this observation.

Indeed, writing $\hat{\mathfrak{n}}_{-}=\mathcal{N}_{-}^{-} \oplus\left(\mathcal{N}_{-}^{+}+\mathcal{H}_{-}\right)$we get $\mathcal{U}\left(\hat{\mathfrak{n}}_{-}\right)=\mathcal{U}\left(\mathcal{N}_{-}^{+}+\mathcal{H}_{-}\right) \oplus \mathcal{N}_{-}^{-} \mathcal{U}\left(\hat{\mathfrak{n}}_{-}\right)$. One has $L(\lambda)=\mathcal{U}\left(\hat{\mathfrak{n}}_{-}\right) \bar{v}_{\lambda}$, where $\bar{v}_{\lambda}$ is the highest weight vector in $L(\lambda)$. By the above observation, $L(\lambda)_{\lambda+\mu}$ does not meet $\mathcal{U}\left(\mathcal{N}_{-}^{+}+\mathcal{H}_{-}\right) \bar{v}_{\lambda}$ and this gives the first inclusion. For the second inclusion, recall that $M^{\prime}(\lambda)=0$ if the level of $\lambda$ is not critical and that $M^{\prime}(\lambda)=\mathcal{U}\left(\hat{\mathfrak{n}}_{-}\right) N$, where $N:=\sum_{j \geq 1} M^{\prime}(\lambda)_{\lambda-j \delta}$ if $\lambda$ has the critical level. By the above observation, the space $\mathcal{U}\left(\mathcal{N}_{-}^{+}+\mathcal{H}_{-}\right) N$ does not meet $M(\lambda)_{\lambda+\mu}$. Hence $M^{\prime}(\lambda)_{\lambda+\mu} \subset \mathcal{N}_{-}^{-} M^{\prime}$ and the second inclusion follows.

Recall that $M(\lambda)$ has a local series at $\lambda+\mu$ with simple quotients $L\left(\lambda-s_{i} \delta\right)$, where $s_{i}$ are non-negative integers. Applying the first formula to the pair $\lambda^{\prime}:=\lambda-s_{i} \delta, \mu^{\prime}:=\mu+s_{i} \delta$ we get $H^{m}\left(\mathfrak{q}, L\left(\lambda-s_{i} \delta\right)\right)_{\lambda+\mu}=0$ for $m=0,1$ and any index $i$. Using the long exact sequence of Lie algebra cohomology we obtain $H^{m}(\mathfrak{q}, M(\lambda))_{\lambda+\mu}=0$.

The proof for $\mathfrak{q}^{-}$are similar.

\subsection{Retain notation of 4.1 .}

Corollary. For $\lambda \in \Lambda$ one has

$$
\begin{array}{ll}
H^{m}\left(\mathfrak{q}_{A}, M(\lambda+x \xi)\right)_{\lambda+x \xi+\mu}=0 & \text { for } m=0,1 \text { and } \mu \in \hat{Q}_{-}^{-}, \\
H^{m}\left(\mathfrak{q}_{A}^{-}, M(\lambda+x \xi)\right)_{\lambda+x \xi+\mu}=0 & \text { for } m=0,1 \text { and } \mu \in \hat{Q}_{+}^{-} .
\end{array}
$$


Proof. Set $R:=\mathbb{C}[x]$ and let $M$ be a Verma $\hat{\mathfrak{g}}_{R}$-module of highest weight $\lambda+x \xi$. Clearly, $M(\lambda+x \xi)=M \otimes_{R} A$. For $c \in \mathbb{C}$ denote by $M(c)$ the evaluation of $M$ at the point $t=c$. Clearly, $M(c)$ is a Verma $\hat{\mathfrak{g}}$-module isomorphic to $M(\lambda+c \xi)$. The choice of $\xi$ ensures that $(\lambda+c \xi) \in \Lambda$ for infinitely many values of $c$. Combining Lemmas 5.2 and 9.2 .1 we obtain the required assertion.

5.4. Let $V_{1}, V_{2}$ be any diagonalizable $\hat{\mathfrak{h}}$-modules, i.e. $V_{i}=\sum_{\xi \in \hat{\mathfrak{h}}^{*}} V_{i, \mu}$. Call a linear map $\varphi: V_{1} \rightarrow V_{2}$ a weight homomorphism if there exists $\nu \in \hat{\mathfrak{h}}^{*}$ such that $\varphi\left(V_{1, \mu}\right) \subset V_{2, \mu+\nu}$ for all $\mu \in \hat{\mathfrak{h}}^{*}$.

5.4.1. Let $W$ be an $(\hat{\mathfrak{h}}+\mathfrak{q})$-module with finite dimensional weight spaces (with respect to $\hat{\mathfrak{h}}$ ) such that for some $\mu \in \hat{\mathfrak{h}}^{*}$ one has $\operatorname{dim} W_{\mu}=1$ and

$$
\Omega(W) \subset\left\{\mu+\nu \mid \nu \in \hat{Q}_{-}^{-} \cup\{0\}\right\} .
$$

Let $w \in W_{\mu}$ be a non-zero vector. Retain notation of 4.1 ,

\subsubsection{Lemma.}

(i) For each weight vector $w^{\prime} \in \breve{M}(\lambda)$ satisfying $\mathfrak{q} w^{\prime}=0$ there exists a unique weight $\mathfrak{q}$-homomorphism $\psi: W \rightarrow M(\lambda)$ such that $\psi(w)=w^{\prime}$.

(ii) For each weight vector $w^{\prime} \in \breve{M}(\lambda+x \xi)$ satisfying $\mathfrak{q}_{A} w^{\prime}=0$ there exists a unique weight $\mathfrak{q}_{A}$-homomorphism $\psi: W_{A} \rightarrow M(\lambda+x \xi)$ such that $\psi(w)=w^{\prime}$.

Proof. Let us prove (ii); the proof of (i) is the same. Recall that $\Omega(\mathfrak{q}) \subset \hat{Q}^{+}$and so the action of $\mathfrak{q}$ raises the weight. As a result, $W$ admits an increasing $(\hat{\mathfrak{h}}+\mathfrak{q})$-filtration $\left\{W^{k}\right\}_{k \geq 0}$ with one-dimensional factors and $W^{0}=W_{\mu}$. Then $\left\{W_{A}^{k}\right\}_{k \geq 0}$ is an increasing $(\hat{\mathfrak{h}}+\mathfrak{q})_{A}$-filtration with factors of rank one over $A$.

Shift the $\hat{\mathfrak{h}}_{A}$-action on $M(\lambda+x \xi)$ by wt $w-\mu$, i.e. set $h . v=(h-($ wt $w-\mu)(h)) v$ for $h \in \hat{\mathfrak{h}}_{A}, v \in M(\lambda+x \xi)$. The shifted action is compatible with the action of $\mathfrak{q}_{A}$; view $M(\lambda+x \xi)$ as $(\hat{\mathfrak{h}}+\mathfrak{q})_{A}$-module with respect to this shifted action. Notice that $\psi$ is an $(\hat{\mathfrak{h}}+\mathfrak{q})_{A}$-homomorphism. In the formulas below we use this shifted $(\hat{\mathfrak{h}}+\mathfrak{q})_{A}$-module structure on $M(\lambda+x \xi)$ and Hom stands for $\operatorname{Hom}_{(\hat{\mathfrak{h}}+\mathfrak{q})_{A}}$.

Let $T^{\nu}$ be a one-dimensional $(\hat{\mathfrak{h}}+\mathfrak{q})$-module of weight $\nu$ (i.e. $\mathfrak{q} T^{\nu}=0,\left.h\right|_{T^{\nu}}=\nu(h)$ id). The short exact sequence

$$
0 \rightarrow W_{A}^{k-1} \rightarrow W_{A}^{k} \rightarrow T_{A}^{\nu} \rightarrow 0
$$

gives

$$
\begin{aligned}
0 & \rightarrow \operatorname{Hom}\left(T_{A}^{\nu}, M(\lambda+x \xi)\right)=H^{0}\left(\mathfrak{q}_{A}, M(\lambda+x \xi)\right)_{\nu^{\prime}} \rightarrow \operatorname{Hom}\left(W_{A}^{k}, M(\lambda+x \xi)\right) \rightarrow \\
& \rightarrow \operatorname{Hom}\left(W_{A}^{k-1}, M(\lambda+x \xi)\right) \rightarrow \operatorname{Ext}^{1}\left(T_{A}^{\nu}, M(\lambda+x \xi)\right)=H^{1}\left(\mathfrak{q}_{A}, M(\lambda+x \xi)\right)_{\nu^{\prime}},
\end{aligned}
$$


where $\nu^{\prime}:=$ wt $w+\nu=\lambda+x \xi-s \delta+\nu$ for some $s \geq 0$. For $k>0$ one has $\nu \in \hat{Q}_{-}^{-}$so either $\nu-s \delta \notin \hat{Q}^{-}$or $\nu-s \delta \in \hat{Q}_{-}^{-}$. Corollary 5.3 gives

$$
H^{0}\left(\mathfrak{q}_{A}, M(\lambda+x \xi)\right)_{\nu^{\prime}}=H^{1}\left(\mathfrak{q}_{A}, M(\lambda+x \xi)\right)_{\nu^{\prime}}=0 .
$$

Therefore $\operatorname{Hom}\left(W_{A}^{k}, M(\lambda+x \xi)\right)=\operatorname{Hom}\left(W_{A}^{k-1}, M(\lambda+x \xi)\right)$. As a result, $\operatorname{Hom}\left(W_{A}^{k}, M(\lambda+\right.$ $x \xi))=\operatorname{Hom}\left(W_{A}^{0}, M(\lambda+x \xi)\right)=\operatorname{Hom}(A w, M(\lambda+x \xi))$. The assertion follows.

5.4.3. The same arguments allow us to deduce the facts similar to Lemma 5.4 .2 for $\mathfrak{q}^{-}$, $\mathfrak{q}_{A}^{-}: \mathfrak{q}$ should be substituted by $\mathfrak{q}^{-}$and $\hat{Q}_{-}^{-}$(in the formula for $\Omega(W)$ ) by $\hat{Q}_{+}^{-}$.

\section{Module $M(\lambda+x \xi)$}

In this section $\lambda \in \Lambda$. We consider a Verma $\hat{\mathfrak{g}}_{A}$-module $M(\lambda+x \xi)$ introduced in 4.1 .2

6.1. Content of the section. We will use the notation $\mathrm{HC}_{ \pm}$for the maps $\mathcal{U}\left(\hat{\mathfrak{n}}_{-}\right)_{A} \rightarrow \mathcal{S}_{A}$ as well as for the corresponding maps $M(\lambda+x \xi) \rightarrow \mathcal{S}_{A}$ and their restrictions. In the following proposition the maps $\mathrm{HC}_{ \pm}: \breve{M}(\lambda+x \xi)^{\mathcal{N}^{ \pm}} \rightarrow \mathcal{S}_{A}$ are the restrictions of $\mathrm{HC}_{ \pm}$: $M(\lambda+x \xi) \rightarrow \mathcal{S}_{A}$

6.1.1. Proposition. For $\lambda \in \Lambda$ the maps $\mathrm{HC}_{+}: \breve{M}(\lambda+x \xi)^{\mathcal{N}^{+}} \stackrel{\sim}{\longrightarrow} \mathcal{S}_{A}, \quad \mathrm{HC}_{-}$: $\breve{M}(\lambda+x \xi)^{\mathcal{N}^{-}} \stackrel{\sim}{\longrightarrow} \mathcal{S}_{A}$ are bijective.

6.1.2. The proposition is proven in 6.3, 6.5, 6.6 below. In 6.3 we explicitly construct $v_{ \pm} \in \breve{M}(\lambda+x \xi)^{\mathcal{N}^{ \pm}}$satisfying $\mathrm{HC}_{ \pm}\left(v_{ \pm}\right)=z$ for each $z \in \mathcal{H}_{-}$. Then in 6.5 we construct such vectors for any $z \in \mathcal{S}$ and thus the surjectivity of the maps $\mathrm{HC}_{ \pm}: \breve{M}(\lambda+x \xi)^{\mathcal{N}^{ \pm}} \rightarrow \mathcal{S}_{A}$ is established. Finally, in 6.6, we show the injectivity of these maps.

6.1.3. Eventhough we are interested in the case $\lambda \in \Lambda_{\text {crit }}$, we consider $\lambda \in \Lambda$. We use this twice: first, the proof of injectivity of $\mathrm{HC}_{ \pm}: \breve{M}(\lambda+x \xi)^{\mathcal{N}^{ \pm}} \rightarrow \mathcal{S}_{A}$ is based on the surjectivity of $\mathrm{HC}_{ \pm}: \breve{M}\left(\lambda^{\prime}+t \xi\right)^{\mathcal{N}^{ \pm}} \rightarrow \mathcal{S}_{A}$, where $t \in \mathbb{C}$ is such that $\lambda^{\prime}=\lambda+t \xi$ lies in $\Lambda \backslash \Lambda_{\text {crit }}$. The second time we use this in Lemma 7.2 , where we prove that, for $\hat{\mathfrak{g}} \neq A(2 k, 2 l)^{(4)}$, the images of vectors $v_{ \pm}$in $M(\lambda)$ are singular if $\lambda \in \Lambda_{\text {crit }}$. The proof of this lemma is based on the results of 6.4, where we study the action of $\mathcal{H}$ on $v_{ \pm}$for $z \in \mathcal{H}_{-}$. We do not use the results of 6.4 in this section and 6.4 is not a part of the proof of Proposition 6.1.1. 
6.2. In 6.3 we will construct $v_{ \pm} \in \breve{M}(\lambda+x \xi)^{\mathcal{N}^{ \pm}}$satisfying $\mathrm{HC}_{ \pm}\left(v_{ \pm}\right)=z$ for $z \in \mathcal{H}_{-}$. Let us describe the construction of $v_{+}$for the non-twisted case (i.e. $\hat{\mathfrak{g}}=\hat{\mathfrak{g}}^{\prime}$ ). The constructions of $v_{-}$is completely similar. The twisted case differs by some technical details.

Take $\lambda \in \Lambda$ and $z=h t^{-m}(h \in \mathfrak{h}, m>0)$. Set

$$
\mathfrak{q}:=\mathfrak{n}+\sum_{s \geq 1}(\mathfrak{h}+\mathfrak{n}) t^{s}, \quad N:=\mathbb{C} h+\sum_{s \geq 0} \mathfrak{n} t^{s}, \quad V:=\mathbb{C} h+\sum_{0 \leq s \leq m-1} \mathfrak{n} t^{s} .
$$

Observe that $N$ is a $\mathfrak{q}$-submodule of $\hat{\mathfrak{g}}$. View $N^{*}$ as a $\mathfrak{q}$-module via the antiautomorphism - id and let $h^{*} \in N^{*}$ be the element dual to $h: h^{*}(h)=1, h^{*}\left(\sum_{s \geq 0} \mathfrak{n} t^{s}\right)=0$. By Lemma 5.4 .2 there exists a unique $\mathfrak{q}_{A}$-homomorphism $\psi: N_{A}^{*} \rightarrow M(\lambda+x \bar{\xi})$ such that $\psi\left(h^{*}\right)$ is the highest weight vector of $M(\lambda+x \xi)$.

For $a \in \mathfrak{g}$ set $T_{m}\left(a t^{s}\right):=a t^{s-m}$. Let $\gamma: \hat{\mathfrak{g}}_{A} \otimes M(\lambda+x \xi) \rightarrow M(\lambda+x \xi)$ be the natural map $\gamma(u \otimes v)=u v$, and let $\operatorname{id}^{\prime} \in V_{A} \otimes N_{A}^{*}$ correspond to the identity map id $\operatorname{c}_{V}$ In Proposition 6.3.5 we prove that the vector

$$
v:=\gamma\left(\left(T_{m} \otimes \psi\right) \mathrm{id}^{\prime}\right) \in M(\lambda+x \xi)
$$

satisfies $\mathrm{HC}_{+}(v)=h t^{-m}$ and $\mathcal{N}^{+} v=0$.

6.3. Fix $\lambda \in \Lambda$ and retain notation of 6.1. Fix $w \in M(\lambda+x \xi)_{\lambda+x \xi-s \delta}$ satisfying $\mathcal{N}^{+} w=0$ and $z=a(-m) \in \mathcal{H}_{-}(m>0)$. In this subsection we will construct vectors $v_{ \pm} \in$ $\breve{M}(\lambda+x \xi)^{\mathcal{N}^{ \pm}}$satisfying $\mathrm{HC}_{ \pm}\left(v_{ \pm}\right)=z \mathrm{HC}_{+}(w)$.

6.3.1. Let $p$ be the reminder of $-m$ modulo $r(0 \leq p \leq r)$.

Let $\mathfrak{g} \neq \mathfrak{g l}(n \mid n)$. Set $T_{m+p}: u(j) \mapsto u(j-p-m)$ for $u \in \mathfrak{g}, j \in \mathbb{Z}$.

Let $\mathfrak{g}=\mathfrak{g l}(n \mid n)$. Define $T_{m+p}$ by the same formula for $j \neq 0, p+m$; set $T_{m+p}(u(0))=$ $\bar{u}(-p-m)$, where $\bar{u}$ is the image of $u \in \mathfrak{g l}(n \mid n)^{\epsilon}$ in $\mathfrak{p g l}(n \mid n)^{\epsilon}$. Finally, for $j=m+p$ choose any linear embedding $\iota: \mathfrak{p g l}(n \mid n) \rightarrow \mathfrak{g l}(n \mid n)$ such that the restriction of $\iota$ to Borel subalgebras containing $\mathfrak{n}$ and $\mathfrak{n}_{-}$are homomorphism; set $T_{m+p}(u(m+p))=\iota(u)(0)$.

From the realization of $\hat{\mathfrak{g}}$ given in 2.3 .3 one sees that $T_{m+p}$ is well-defined linear endomorphism of the space generated by the elements $u(j)$; this space is the orthogonal compliment of $(\mathbb{C} K+\mathbb{C} D)$ in $\hat{\mathfrak{g}}$ with respect to the form $(-\mid-)$. Observe that

$$
T_{m+p}([x, y])=\left[x, T_{m+p}(y)\right]=\left[T_{m+p}(x), y\right] \quad \text { if wt } x+\text { wt } y \notin\{0,(m+p) \delta\} .
$$

Fix $a(p) \in \tilde{\mathcal{H}}$ such that

$$
T_{m+p}(a(p))=a(-m)
$$

this element is unique unless $\mathfrak{g}=\mathfrak{g l}(n \mid n), p=0$.

6.3.2. Set $\mathcal{H}(z):=\{u \in \mathcal{H} \mid[u, a(p)]=0\}$ and fix a subalgebra $\mathfrak{q} \subset \hat{\mathfrak{g}}$ such that

$$
\mathcal{N}^{+} \subset \mathfrak{q} \subset \mathcal{N}^{+}+\mathcal{H}(z)
$$

For $\hat{\mathfrak{g}} \neq A(2 k, 2 l)^{(4)}$ one has $\mathcal{H}(z)=\mathcal{H}$ for all $z$. 
6.3.3. Set $N:=\mathbb{C} a(p)+\sum_{s \geq p} \mathcal{N}_{s}^{+}$and write $N=V \oplus\left(\sum_{s \geq m+p} \mathcal{N}_{s}^{+}\right)$, where

$$
V:=\mathbb{C} a(p)+\sum_{p \leq s<m+p} \mathcal{N}_{s}^{+} .
$$

For the non-twisted case $(r=1)$ one has $p=0$ and $N=\mathbb{C} a(0)+\sum_{s \geq 1} \mathfrak{n} t^{s}$.

Set

$$
V^{*}:=\left\{f \in \operatorname{Hom}(N, \mathbb{C}) \mid f\left(\sum_{s \geq m+p} \mathcal{N}_{s}^{+}\right)=0\right\} .
$$

Both $N, \sum_{s \geq m+p} \mathcal{N}_{s}^{+}$are $(\hat{\mathfrak{h}}+\mathfrak{q})$-modules with respect to the adjoint action; view $N^{*}, V^{*}$ as $(\hat{\mathfrak{h}}+\mathfrak{q})$-modules via the antiautomorphism - id. Observe that $V$ and $V^{*}$ are dual $\hat{\mathfrak{h}}$-modules. Let $a(p)^{*} \in V^{*}$ be the "dual to $a(p)$ ", that is

$$
a(p)^{*}(a(p))=1, \quad \forall u \in \sum_{s \geq p} \mathcal{N}_{s}^{+} a(p)^{*}(u)=0 .
$$

6.3.4. Let $w \in \breve{M}(\lambda+x \xi)$ be a weight vector satisfying $\mathfrak{q}_{A} w=0$. By Lemma 5.4.2 (ii) there exists a unique weight $\mathfrak{q}_{A}$-homomorphism $\psi: V_{A}^{*} \rightarrow M(\lambda+x \xi)$ such that $\psi\left(a(p)^{*}\right)=w$.

6.3.5. Proposition. Let $\gamma: \hat{\mathfrak{g}}_{A} \otimes M(\lambda+x \xi) \rightarrow M(\lambda+x \xi)$ be the natural map $\gamma(u \otimes w):=u w$, and let $\operatorname{id}^{\prime} \in V_{A} \otimes V_{A}^{*}$ correspond to the identity map $V_{A} \rightarrow V_{A}$. Then

$$
v:=\gamma\left(\left(T_{m+p} \otimes \psi\right) \mathrm{id}^{\prime}\right) \in M(\lambda+x \xi)
$$

is a weight vector such that $\mathrm{HC}_{+}(v)=a(-m) \mathrm{HC}_{+}(w)$ and $u v=0$ for any weight element $u \in \mathfrak{q}_{A}$ if its weight is not equal to $m \delta$.

Proof. Let $B$ be a weight basis of $V \cap \mathcal{N}^{+}$; then $B^{\prime}:=\{a(p)\} \cup B$ is a weight basis of $V$. For $b \in B$ denote by $b^{*}$ the element of the dual basis $\left\{a(p)^{*}\right\} \cup B^{*}$ of $V^{*}$. Then

$$
v=\sum_{b \in B^{\prime}} T_{m+p}(b) \psi\left(b^{*}\right)=a(-m) w+\sum_{b \in B} T_{m+p}(b) \psi\left(b^{*}\right) .
$$

Notice that $T_{m+p}(b) \in \mathcal{N}_{-}^{+}$and so $\mathrm{HC}_{+}(v)=a(-m) \mathrm{HC}_{+}(w)$ as required.

It remains to show that $u v=0$ if wt $u \neq m \delta$. Fix such element $u$. Since $\psi$ is $\mathfrak{q}_{A^{-}}$-invariant we have

$$
u v=\sum_{b \in B^{\prime}} u T_{m+p}(b) \psi\left(b^{*}\right)=\sum_{b \in B^{\prime}}\left[u, T_{m+p}(b)\right] \psi\left(b^{*}\right)+(-1)^{p(u) p(b)} T_{m+p}(b) \psi\left(u b^{*}\right) .
$$

Since wt $u \neq m \delta$ the formula (9) implies $T_{m+p}([u, x])=\left[u, T_{m+p}(x)\right]$. Therefore

$$
u v=\sum_{b \in B^{\prime}} T_{m+p}([u, b]) \psi\left(b^{*}\right)+(-1)^{p(u) p(b)} T_{m+p}(b) \psi\left(u b^{*}\right) .
$$


For $b_{s} \in B^{\prime}$ write

$$
\left[u, b_{s}\right]=\sum_{b_{j} \in B^{\prime}} c_{s j} b_{j}+w_{s}, \quad \text { where } c_{s j} \in \mathbb{C}, w_{s} \in \sum_{t \geq m+p} \mathcal{N}_{t}^{+} .
$$

Then $u b_{s}^{*}=(-1)^{p(u) p\left(b_{s}^{*}\right)+1} \sum c_{j s} b_{j}^{*}$ and this gives $u v=\sum_{s} T_{m+p}\left(w_{s}\right) \psi\left(b_{s}^{*}\right)$. Observe that $T_{m+p}\left(w_{s}\right) \in \mathcal{N}^{+} ;$since $\psi$ is $\mathcal{N}^{+}$-invariant we obtain

$$
u v=\psi\left(\sum_{s} T_{m+p}\left(w_{s}\right) b_{s}^{*}\right) .
$$

We claim that $T_{m+p}\left(w_{s}\right) b_{s}^{*}=0$. Indeed, wt $w_{s}=\mathrm{wt} u+\mathrm{wt} b_{s}$ so the weight of $T_{m+p}\left(w_{s}\right) b_{s}^{*}$ is wt $u-(m+p) \delta$. However $(\operatorname{wt} u-(m+p) \delta) \notin \Omega\left(V_{A}^{*}\right)$ because $\Omega\left(V_{A}^{*}\right)=-\Omega(V) \subset$ $\Omega\left(\mathcal{N}_{-}^{-}\right) \cup\{-p \delta\}$. Hence $T_{m+p}\left(w_{s}\right) b_{s}^{*}=0$. This completes the proof.

6.3.6. Remark. Fix a subalgebra $\mathfrak{q}^{-}$of $\hat{\mathfrak{g}}$ such that

$$
\mathcal{N}^{-} \subset \mathfrak{q}^{-} \subset \mathcal{N}^{-}+\mathcal{H}(z)
$$

and fix a weight vector $w_{-} \in \breve{M}(\lambda+x \xi)^{\mathfrak{q}^{-}}$.

Substituting $\mathfrak{q}$ by $\mathfrak{q}^{-}, w$ by $w_{-}, N$ by $N^{-}:=\mathbb{C} a(p)+\sum_{s>p} \mathcal{N}_{s}^{-}$and $V$ by $V^{-}:=$ $\mathbb{C} a(p)+\sum_{p<s \leq m+p} \mathcal{N}_{s}^{+}$, we obtain a weight vector $v \in M(\lambda+x \xi)$ which satisfies $\mathrm{HC}_{-}(v)=$ $z \mathrm{HC}_{-}\left(w_{-}\right)$and $u v=0$ for any $u \in \mathfrak{q}^{-}$provided that wt $u \neq m \delta$.

6.4. Let $v_{\lambda+x \xi}$ be the canonical generator of $M(\lambda+x \xi)$. Retain notation of Proposition 6.3 .5 and construct $v$ for $w:=v_{\lambda+x \xi}$. The following lemmas will be used later.

\subsubsection{Lemma.}

(i) Take $0<j<m$. For any $d(j) \in \mathcal{H}_{j}$ one has

$$
\mathrm{HC}_{+}(d(j) v)=[d(j), a(-m)] v_{\lambda+x \xi} ;
$$

(ii) For any $d(m) \in \mathcal{H}_{m}$ satisfying $[d(m), a(p)]=0$ one has

$$
\begin{aligned}
& d(m) v=([d(m), a(-m)]-c) v_{\lambda+x \xi}, \quad \text { where } \\
& c:=\sum_{p \leq s<m+p} \frac{1}{2} \operatorname{str}_{\hat{\mathfrak{g}}_{s}}(\operatorname{ad} d(-p) \circ \operatorname{ad} a(p))-\operatorname{str}_{\tilde{\mathcal{H}}_{s}}(\operatorname{ad} d(-p) \circ \operatorname{ad} a(p)) .
\end{aligned}
$$

Proof. Recall that

$$
v=a(-m) v_{\lambda+x \xi}+\sum_{b \in B} T_{m+p}(b) \psi\left(b^{*}\right),
$$

where $B \cup\{a(p)\}$ is a weight basis of $V=\mathbb{C} a(p)+\sum_{p \leq s<m+p} \mathcal{N}_{s}^{+}$. For (i) write

$$
d(j) v=[d(j), a(-m)] v_{\lambda+x \xi}+\sum_{b \in B}\left[d(j), T_{m+p}(b)\right] \psi\left(b^{*}\right)+\sum_{b \in B} \pm T_{m+p}(b)\left(d(j) \psi\left(b^{*}\right)\right) .
$$


Since $T_{m+p}(b) \in \mathcal{N}_{-}^{+}$the term $T_{m+p}(b)\left(d(j) \psi\left(b^{*}\right)\right)$ lies in the kernel of $\mathrm{HC}_{+}$. Moreover, if $b$ is such that $\left[d(j), T_{m+p}(b)\right] \in \mathcal{N}_{-}^{+}$then $\left[d(j), T_{m+p}(b)\right] \psi\left(b^{*}\right) \in \mathrm{Ker} \mathrm{HC}_{+}$. Otherwise $\left[d(j), T_{m+p}(b)\right] \in \mathcal{N}^{+}$so

$$
\left[d(j), T_{m+p}(b)\right] \psi\left(b^{*}\right)=\psi\left(\left[d(j), T_{m+p}(b)\right] b^{*}\right) .
$$

The term $\left[d(j), T_{m+p}(b)\right] b^{*}$ has weight $-(m+p-j) \delta$ so it is equal to zero (because $\left.\Omega\left(V^{*}\right) \cap \mathbb{Z} \delta=\{-p \delta\}\right)$. This proves (i).

For (ii) notice that $d(m) V^{*}=0$ since there are no $\mu, \mu^{\prime} \in \Omega\left(V^{*}\right)$ satisfying $\mu-\mu^{\prime}=m \delta$. Since $d(m) \in \mathfrak{q}$ this gives $d(m) \psi\left(b^{*}\right)=0$ for any $b \in B$. Therefore

$$
d(m) v=[d(m), a(-m)] v_{\lambda+x \xi}+\sum_{b \in B}\left[d(m), T_{m+p}(b)\right] \psi\left(b^{*}\right) .
$$

Recall that $b \in \mathcal{N}^{+}$and so

$$
\left[d(m), T_{m+p}(b)\right]=\left[T_{m+p}(d(m)), b\right]=[d(-p), b] \in \mathcal{N}^{+} .
$$

Now we can rewrite the expression for $d(m) v$ in the form

$$
d(m) v=[d(m), a(-m)] v_{\lambda+x \xi}+\psi\left(\sum_{b \in B}[d(-p), b] b^{*}\right) .
$$

Since $M(\lambda+x \xi)$ is one-dimensional, $d(m) v=0$ if $d(m)$ and $a(-m)$ have different parities. Note that the parities of $a(p)$ and $a(-m)$ are equal. Therefore (ii) holds if the parities of $d(m)$ and $a(p)$ are not equal. Hence we may (and will) assume that $d(m)$ and $a(p)$ have the same parity.

The term $[d(-p), b] b^{*}$ has weight $-p \delta$ and so $[d(-p), b] b^{*}=c_{b} a(p)^{*}$ for some scalar $c_{b}$. Since $d(m), a(p)$ have the same parity we obtain

$$
c_{b}=\left([d(-p), b] b^{*}\right)(a(p))=(-1)^{1+p(b)} b^{*}([d(-p),[a(p), b]]) .
$$

Since $\psi\left(a(p)^{*}\right)=v_{\lambda+x \xi}$ one has

$$
d(m) v=X v_{\lambda+x \xi}, \text { where } X:=[d(m), a(-m)]+\sum_{b \in B}(-1)^{1+p(b)} b^{*}([d(-p),[a(p), b]]) .
$$

Notice that $B$ spans $V \cap \mathcal{N}^{+}$so

$$
X=[d(m), a(-m)]-\operatorname{str}_{V \cap \mathcal{N}^{+}}(\operatorname{ad} d(-p) \circ \operatorname{ad} a(p)) .
$$

Clearly, $V \cap \mathcal{N}^{+}=\sum_{p \leq s<m+p} \mathcal{N}_{s}^{+}$. Recall (see 2.6.1) that $\hat{\mathfrak{g}}$ admits an automorphism $\varepsilon$ satisfying $\varepsilon\left(\tilde{\mathcal{N}}_{j}^{+}\right)=\tilde{\mathcal{N}}_{j}^{-}$which stabilizes the product $d(-p) a(p)$. Therefore $\operatorname{str}_{\mathcal{N}_{j}^{+}}(\operatorname{ad} d(-p) \circ \operatorname{ad} a(p))=\operatorname{str}_{\mathcal{N}_{j}^{-}}(\operatorname{ad} d(-p) \circ \operatorname{ad} a(p))$. Hence

$$
\operatorname{str}_{V \cap N^{+}}(\operatorname{ad} d(-p) \circ \operatorname{ad} a(p))=\frac{1}{2} \operatorname{str}_{W}(\operatorname{ad} d(-p) \circ \operatorname{ad} a(p))-\operatorname{str}_{W \cap \tilde{\mathcal{H}}}(\operatorname{ad} d(-p) \circ \operatorname{ad} a(p)),
$$

where $W:=\sum_{p \leq j<m+p} \hat{\mathfrak{g}}_{j}$. This proves (ii).

6.4.2. A lemma similar to Lemma 6.4.1 holds for $\mathrm{HC}_{-}$. 
MARIA GORELIK

6.5. Proof of surjectivity in Proposition 6.1.1. Take any vector weight vector $w_{1} \in$ $\breve{M}(\lambda+x \xi)^{\mathcal{N}^{+}}$. Applying Proposition 6.3.5 for $\mathfrak{q}=\mathcal{N}^{+}$and $w:=w_{1}$ we obtain $w_{2} \in$ $\breve{M}(\lambda+x \xi)^{\mathcal{N}^{+}}$satisfying $\mathrm{HC}_{+}\left(w_{2}\right)=a(-m) \mathrm{HC}_{+}\left(w_{1}\right)$. Since the elements of the form $a(-m)$ generate $\mathcal{S}_{A}$, the surjectivity follows.

6.6. Proof of injectivity in Proposition 6.1.1. Let us show that the restrictions of $\mathrm{HC}_{ \pm}$to $\breve{M}(\lambda)^{\mathcal{N}^{ \pm}}$are injective.

Observe that for any $\lambda^{\prime} \in \hat{\mathfrak{h}}$ the space $\breve{M}\left(\lambda^{\prime}\right)^{\mathcal{N}^{+}}$is orthogonal to Ker HC_ $\cap \breve{M}\left(\lambda^{\prime}\right)$ with respect to the Shapovalov form, see (4). If $\lambda^{\prime}$ is such that $M\left(\lambda^{\prime}\right)$ is irreducible then its Shapovalov form is non-degenerate and thus

$$
\operatorname{ch} \breve{M}\left(\lambda^{\prime}\right)^{\mathcal{N}^{+}} \leq e^{\lambda^{\prime}} \operatorname{ch} \mathcal{S}
$$

Now take $\lambda^{\prime} \in \Lambda$. Then $\mathrm{HC}_{+}: \breve{M}\left(\lambda^{\prime}+x \xi\right)^{\mathcal{N}^{+}} \rightarrow \mathcal{S}_{A}$ is surjective so $\mathrm{HC}_{+}: \breve{M}\left(\lambda^{\prime}\right)^{\mathcal{N}^{+}} \rightarrow \mathcal{S}$ is also surjective. The above inequality gives

$$
\mathrm{HC}_{+}: \breve{M}\left(\lambda^{\prime}\right)^{\mathcal{N}^{+}} \stackrel{\sim}{\longrightarrow} \mathcal{S} \quad \text { if } M\left(\lambda^{\prime}\right) \text { is irreducible. }
$$

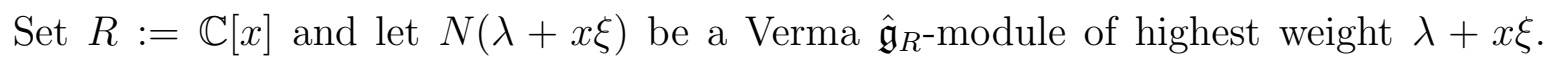
Clearly, $M(\lambda+x \xi)=N(\lambda+x \xi) \otimes_{R} A$. Identify $N(\lambda+x \xi)$ with the corresponding $R$ submodule of $M(\lambda+x \xi)$. Assume that $\mathrm{HC}_{+}: \breve{M}(\lambda+x \xi)^{\mathcal{N}^{+}} \rightarrow \mathcal{S}_{A}$ is not injective. Then there exists a non-zero $v \in \breve{N}(\lambda+x \xi)^{\mathcal{N}^{+}}$such that $\mathrm{HC}_{+}(v)=0$. Note that the evaluation of $N(\lambda+x \xi)$ at $x=t \in \mathbb{C}$ is a Verma $\hat{\mathfrak{g}}$-module $M(\lambda+t \xi)$. The choice of $\xi$ ensures that $M(\lambda+t \xi)$ is irreducible for $t \in \mathbb{C} \backslash Y$, where $Y$ is at most countable. The image of any non-zero vector $v^{\prime} \in N(\lambda+x \xi)$ in the evaluated module $M(\lambda+t \xi)$ is zero only for finitely many values of $t \in \mathbb{C}$. Hence for some $t \in \mathbb{C} \backslash Y$ the image $\bar{v}$ of $v$ in $M(\lambda+t \xi)$ is non-zero. This contradicts to (11), since $M(\lambda+t \xi)$ is irreducible, $\mathcal{N}^{+} \bar{v}=0$ and $\mathrm{HC}_{+}(\bar{v})=0$. This establishes the injectivity and completes the proof of Proposition 6.1.1.

\section{Proof of 3.2, (A)}

In this section $\hat{\mathfrak{g}}$ is not of the type $A(2 k, 2 l)^{(4)}$.

The assertion (A) of 3.2 follows from Proposition 7.3 and Proposition 7.4 below.

7.1. Notation. By Proposition 6.1.1 the map $\mathrm{HC}_{+}: \breve{M}(\lambda+x \xi)^{\mathcal{N}^{+}} \rightarrow \mathcal{S}_{A}$ is bijective. Let $\mathrm{HC}_{+}^{-1, \lambda}: \mathcal{S} \rightarrow M(\lambda)$ be the evaluation of the inverse map $\mathcal{S}_{A} \rightarrow \breve{M}(\lambda+x \xi)^{\mathcal{N}^{+}}$at the point $x=0$. Clearly, $\mathrm{HC}_{+} \circ \mathrm{HC}_{+}^{-1, \lambda}=\mathrm{id}_{\mathcal{S}}$. Define similarly the map $\mathrm{HC}_{-}^{-1, \lambda}: \mathcal{S} \rightarrow M(\lambda)$.

7.2. We start from the following lemma.

Lemma. Take $\lambda \in \Lambda_{\text {crit }}$. For any $u \in \mathcal{H}_{-}$the vectors $\operatorname{HC}_{+}^{-1, \lambda}(u), \operatorname{HC}_{-}^{-1, \lambda}(u) \in M(\lambda)$ are singular. 
Proof. We will prove that $\mathrm{HC}_{+}^{-1, \lambda}(u)$ is singular; the proof for $\mathrm{HC}_{-}^{-1, \lambda}(u)$ is similar. We may (and will) assume that $u$ is of the form $u=a(-m)$ for some $m>0$.

Fix any $\lambda^{\prime} \in \Lambda$ and let $v_{\lambda^{\prime}+x \xi}$ be the canonical generator of $M\left(\lambda^{\prime}+x \xi\right)$. Let $v^{\prime} \in$ $\breve{M}\left(\lambda^{\prime}+x \xi\right)$ be a vector satisfying $\mathcal{N}^{+} v^{\prime}=0, \quad \mathrm{HC}_{+}\left(v^{\prime}\right)=a(-m)$. By 6.1.1, $v^{\prime}$ is unique and so it coincides with the vector constructing in Proposition 6.3.5 for $w=v_{\lambda^{\prime}+x \xi}$. Recall that $\mathcal{H}$ is commutative (see 2.5) and so in 6.3.5 we may choose $\mathfrak{q}=\mathcal{N}^{+}+\mathcal{H}$. Then 6.3.5 gives $\mathcal{H}_{s} v^{\prime}=0$ for $s<m$. Clearly, $\mathcal{H}_{s} v^{\prime}=0$ for $s>m$.

Let $\bar{v}^{\prime}$ be the image of $v^{\prime}$ at $M\left(\lambda^{\prime}\right)$. By definition, $\mathrm{HC}_{+}^{-1, \lambda^{\prime}}(u)=\bar{v}^{\prime}$. Notice that $\bar{v}^{\prime} \in \breve{M}\left(\lambda^{\prime}\right)$ and $\mathcal{N}^{+} \bar{v}^{\prime}=\mathcal{H}_{s} v^{\prime}=0$ for $s \neq m$. In the light of Lemma 2.12.1, $\bar{v}^{\prime}$ is singular if $\mathcal{H}_{m} \bar{v}=0$. Take any $d(m) \in \mathcal{H}_{m}$.

Recall that $\mathcal{H}$ is commutative. Combining Lemma 2.7 and Lemma 6.4.1 (ii) we get $d(m) v^{\prime}=X v_{\lambda^{\prime}+x \xi}$, where

$$
X=m(d(m) \mid a(-m)) K-\frac{1}{2} \operatorname{str}_{W}(\operatorname{ad} d(-p) \circ \operatorname{ad} a(p)), \quad \text { for } W:=\sum_{p \leq j<m+p} \hat{\mathfrak{g}}_{j} .
$$

The restriction of $(-\mid-)$ to $\hat{\mathfrak{g}}_{m} \otimes \hat{\mathfrak{g}}_{-m}$ is a non-degenerate pairing, which is invariant with respect to the adjoint action of $\hat{\mathfrak{g}}_{0}$. Note that the pairing $B^{\prime}: \hat{\mathfrak{g}}_{-p} \otimes \hat{\mathfrak{g}}_{p} \rightarrow \mathbb{C}$ given by $B^{\prime}(x \mid y):=\operatorname{str}_{W}(\operatorname{ad} x \circ$ ad $y)$ is also invariant with respect to the adjoint action of $\hat{\mathfrak{g}}_{0}$. Identify $\hat{\mathfrak{g}}_{-m}\left(\right.$ resp., $\left.\hat{\mathfrak{g}}_{m}\right)$ with its image $T_{-m-p}\left(\hat{\mathfrak{g}}_{-m}\right) \subset \hat{\mathfrak{g}}_{p}\left(\operatorname{resp} ., T_{m+p}\left(\hat{\mathfrak{g}}_{m}\right) \subset \hat{\mathfrak{g}}_{-p}\right)$ via $T_{-m-p}$ (resp., via $T_{m+p}$ ). Observe that $\hat{\mathfrak{g}}_{m}$ is a simple $\hat{\mathfrak{g}}_{0}$-module (see [K2] and [vdL], 6.10). Thus $B^{\prime}$ is proportional to the restriction of $(-\mid-)$; let $c$ be the coefficient of proportionality. Note that $c$ does not depend on $\lambda^{\prime}$.

We obtain $X=(d(m) \mid a(-m))(m K+c)$, that is

$$
d(m) \bar{v}^{\prime}=(d(m) \mid a(-m))(m K+c) v_{\lambda^{\prime}}=(d(m) \mid a(-m))\left(m\left(\lambda^{\prime}, \delta\right)+c\right) v_{\lambda^{\prime}} .
$$

Therefore $\bar{v}^{\prime}$ is singular if $\lambda$ has level $-c / m$. Since $\mathrm{HC}_{+}\left(\bar{v}^{\prime}\right)=a(-m)$, the vector $\bar{v}^{\prime}$ is non-zero for all $\lambda^{\prime} \in \Lambda$. Recall that $M\left(\lambda^{\prime}\right)$ is simple if $\lambda^{\prime}$ has a non-critical level. Thus $\bar{v}^{\prime}$ is not singular if the level is non-critical. Hence $\bar{v}^{\prime}$ is singular if $\lambda^{\prime}$ has the critical level.

7.3. Proposition. For $\lambda \in \Lambda_{\text {crit }}$ the images of $\mathrm{HC}_{ \pm}^{-1, \lambda}: \mathcal{S} \rightarrow M(\lambda)$ lie in $M(\lambda)^{\hat{\mathfrak{n}}}$.

Proof. We will prove that $\mathrm{HC}_{+}^{-1, \lambda}(z)$ is singular for $z \in \mathcal{S}$; the proof for $\mathrm{HC}_{-}^{-1, \lambda}(z)$ is similar.

For $v \in M(\lambda+x \xi)$ denote by $\bar{v}$ its image in $M(\lambda)$. By Proposition 6.1.1 the map $\mathrm{HC}_{+}: \breve{M}(\lambda+x \xi)^{\mathcal{N}^{+}} \rightarrow \mathcal{S}_{A}$ is bijective; denote by $\mathrm{HC}_{+}^{-1, \lambda+x \xi}$ the inverse map.

For $z \in \mathcal{H}_{-}$the assertion is proven in Lemma 7.2. Since $\mathcal{S}$ is generated by $\mathcal{H}_{-}$, it is enough to show that

$$
\mathrm{HC}_{+}^{-1, \lambda}(z) \text { is singular } \Longrightarrow \mathrm{HC}_{+}^{-1, \lambda}(a(-m) z) \text { is singular . }
$$


Set $z_{1}:=a(-m), z_{2}:=z, z_{3}=a(-m) z$ and let $w_{i}:=\operatorname{HC}_{+}^{-1, \lambda+x \xi}\left(z_{i}\right)$ for $i=1,2,3$. The above implication takes form: $\mathfrak{\mathfrak { n }} \bar{w}_{2}=0 \Longrightarrow \hat{\mathfrak{n}} \bar{w}_{3}=0$. Assume that $\hat{\mathfrak{n}} \bar{w}_{2}=0$. Let $\phi \in \operatorname{End}_{[\hat{\mathfrak{g}}, \hat{\mathfrak{g}}]}(M(\lambda))$ be such that $\phi\left(v_{\lambda}\right)=\bar{w}_{2}$ (see 3.1.1). We claim that

$$
\bar{w}_{3}=\phi\left(\bar{w}_{1}\right) .
$$

By Lemma $7.2 \bar{w}_{1}$ is singular so the formula (12) implies the required implication.

Let us prove (12). Set $\mathfrak{q}:=\mathcal{N}^{+}$. Apply the construction described in 6.3 for $w:=$ $v_{\lambda+x \xi}$ (resp., for $w:=w_{2}$ ) and denote by $\psi_{1}$ (resp., by $\psi_{2}$ ) the weight $\mathfrak{q}_{A}$-homomorphism introduced in 6.3.4. By constructions of $w_{1}$ (Proposition 6.3.5) and of $w_{3}$ (6.5) one has

$$
w_{1}=\sum_{b \in B^{\prime}} T_{m+p}(b) \psi_{1}\left(b^{*}\right), \quad w_{3}=\sum_{b \in B^{\prime}} T_{m+p}(b) \psi_{2}\left(b^{*}\right)
$$

For $i=1,2$ denote by $\psi_{i}(0): V^{*} \rightarrow M(\lambda)$ the evaluation of $\psi_{i}$ at $x=0$; clearly, $\psi_{i}(0)$ is a $\mathfrak{q}$-homomorphism. One has

$$
\psi_{1}\left(a(p)^{*}\right)=v_{\lambda+x \xi}, \quad \phi\left(v_{\lambda}\right)=\bar{w}_{2}, \quad \psi_{2}\left(a(p)^{*}\right)=w_{2}
$$

so $\phi\left(\psi_{1}(0)\left(a(p)^{*}\right)\right)=\psi_{2}(0)\left(a(p)^{*}\right)$. Since both $\phi \circ \psi_{1}(0)$ and $\psi_{2}(0)$ are weight $\mathfrak{q}$-homomorphisms $V^{*} \rightarrow M(\lambda)$, the uniqueness proven in Lemma 5.4 .2 (i) gives

$$
\phi \circ \psi_{1}(0)=\psi_{2}(0) \text {. }
$$

Now (13) gives

$$
\bar{w}_{3}=\sum_{b \in B^{\prime}} T_{m+p}(b) \phi\left(\psi_{1}(0)\left(b^{*}\right)\right)=\phi\left(\sum_{b \in B^{\prime}} T_{m+p}(b) \psi_{1}(0)\left(b^{*}\right)\right)=\phi\left(\bar{w}_{1}\right) .
$$

This establishes (12) and completes the proof.

7.4. Proposition. Take $\lambda \in \Lambda_{\text {crit }}$. For $v \in \breve{M}(\lambda+x \xi)^{\mathcal{N}^{ \pm}}$one has $v \in \mathcal{F}^{k}(M(\lambda+x \xi))$, where $k$ is the degree of $\mathrm{HC}_{ \pm}(v)$.

Proof. The assertions for $\mathcal{N}^{+}$and for $\mathcal{N}^{-}$have similar proofs. Recall that $S_{A}(\lambda+x \xi)$ is the Shapovalov form on $M(\lambda+x \xi)$; denote this form by $(-,-)$. We will prove that

for $v_{+} \in \breve{M}(\lambda+x \xi)^{\mathcal{N}^{+}}$and $v \in M(\lambda+x \xi)$ one has $\left(v_{+}, v\right) \in(x)^{k}$, where $k$ is the degree of $\mathrm{HC}_{+}\left(v_{+}\right)$.

The proof is by induction on $k$. For $k=0$ the assertion is trivial.

Let $v_{-} \in M(\lambda+x \xi)^{\mathcal{N}^{-}}$be such that $\mathrm{HC}_{-}\left(v_{-}\right)=\mathrm{HC}_{-}(v)$. Similarly to (41) one has $\left(v_{+}\right.$, Ker HC-$)=\left(\right.$Ker $\left.\mathrm{HC}_{+}, v_{-}\right)=0$. Therefore

$$
\left(v_{+}, v\right)=\left(v_{+}, v_{-}\right)=\left(\mathrm{HC}_{+}\left(v_{+}\right) v_{\lambda+x \xi}, v_{-}\right)
$$

We may (and will) assume that $z:=\mathrm{HC}_{+}\left(v_{+}\right)$is of the form $z=u w$ for $u \in \mathcal{H}_{-}, w \in \mathcal{S}$. Then

$$
\left(\mathrm{HC}_{+}\left(v_{+}\right) v_{\lambda+x \xi}, v_{-}\right)=\left(z v_{\lambda+x \xi}, v_{-}\right)=\left(w v_{\lambda+x \xi}, \sigma(u) v_{-}\right)
$$


Since $\sigma(u) \in \hat{\mathfrak{n}}$, Proposition 7.3 gives $\sigma(u) v_{-}=x v^{\prime}$ for some $v^{\prime} \in M(\lambda+x \xi)$. Moreover, since $\sigma(u) \in \mathcal{H}$ one has $\mathcal{N}^{-}\left(\sigma(u) v_{-}\right) \subset \mathcal{N}^{-} v_{-}=0$ so $\mathcal{N}^{-} v^{\prime}=0$. Thus

$$
\left(v_{+}, v\right)=x\left(w v_{\lambda+x \xi}, v^{\prime}\right)
$$

for $v^{\prime} \in \breve{M}(\lambda+x \xi)^{\mathcal{N}^{-}}$. Taking $w_{+} \in \breve{M}(\lambda+x \xi)^{\mathcal{N}^{+}}$such that $\mathrm{HC}_{+}\left(w_{+}\right)=w$ we obtain

$$
\left(v_{+}, v\right)=x\left(w_{+}, v^{\prime}\right) .
$$

Notice that $w=\mathrm{HC}_{+}\left(w_{+}\right)$is a monomial of degree $k-1$. By induction hypothesis, $\left(w_{+}, v^{\prime}\right) \in(x)^{k-1}$. This completes the proof.

7.5. Combining Propositions 7.3 and 7.4 we obtain that $\operatorname{HC}_{ \pm}^{-1, \lambda}(\mathcal{S} \geq k)$ lies in $M(\lambda)^{k} \cap$ $M(\lambda)^{\hat{\mathfrak{n}}}$. Hence $\mathrm{HC}_{ \pm}\left(M(\lambda)^{k} \cap M(\lambda)^{\hat{\mathfrak{n}}}\right)$ contains $\mathcal{S}^{\geq k}$ and this establishes $3.2(\mathrm{~A})$.

\section{Proof of 3.2 , (B)}

In this section $\hat{\mathfrak{g}}$ is not of the type $A(2 k, 2 l)^{(4)}$ and $\lambda \in \Lambda_{\text {crit }}$.

In this section we prove the assertion (B) of 3.2. As it is shown in Sect. 3 this completes the proof of Theorems 1.1.1, 1.1.3.

\subsection{Notation. Set}

$$
Z:=\frac{\operatorname{ch} \mathcal{U}\left(\hat{\mathfrak{n}}_{-}\right)}{\operatorname{ch} \mathcal{S}}=\operatorname{ch} \mathcal{U}\left(\mathcal{N}_{-}^{-}\right) \operatorname{ch} \mathcal{U}\left(\mathcal{N}_{-}^{+}\right)
$$

In 2.11.1 we introduced a ring $C$ which contains, in particularly, ch $M(\lambda)$, ch $L(\lambda)$ and $Z$. Introduce the following projections $P_{\nu}, P_{\geq \nu}: C \rightarrow C$ :

$$
P_{\nu}\left(\sum c_{\mu} e^{\mu}\right):=c_{\nu} e^{\nu}, \quad P_{>\nu}\left(\sum c_{\mu} e^{\mu}\right):=\sum_{\mu>\nu} c_{\mu} e^{\mu} .
$$

Set $\operatorname{ch}_{>\nu} N:=P_{>\nu}(\operatorname{ch} N)$.

Denote by $a_{k, s}$ the multiplicity of $L(\lambda-s \delta)$ in $M(\lambda)^{k}$ :

$$
a_{k, s}:=\left[M(\lambda)^{k}: L(\lambda-s \delta)\right]
$$

8.2. Recall that $\left\{\mathcal{S}^{\geq k}\right\}$ is the adic filtration on $\mathcal{S}$. We will prove by induction on $m \geq 0$ that

(i) $\quad a_{k, j}=\operatorname{dim} \mathcal{S}_{-j \delta}^{\geq k}$ for $j=0, \ldots, m-1$ and all $k$;

(ii) $\operatorname{ch}_{>\lambda-m \delta} L(\lambda)=e^{\lambda} P_{>-m \delta}(Z)$.

Clearly, 3.2 (B) follows from (i) for $m=1,2, \ldots$. 
8.2.1. For $m=1$ (i) holds because $a_{k, 0}=\delta_{k, 0}=\operatorname{dim} \mathcal{S}_{0}^{\geq k}$. For (ii) recall that $[M(\lambda)$ : $L(\mu)]=0$ for $\mu>\lambda-\delta$ so $\operatorname{ch}_{>\lambda-\delta} L(\lambda)=\operatorname{ch}_{>\lambda-\delta} M(\lambda)$. Therefore

$$
\operatorname{ch}_{>\lambda-\delta} L(\lambda)=e^{\lambda} P_{>-\delta}(\mathcal{S} \cdot Z)=e^{\lambda} P_{>-\delta}(Z),
$$

since $P_{>-\delta}(\mathcal{S})=1$ and $P_{\nu}(Z)=0$ for $\nu \not \leq 0$.

8.2.2. Assume that (14) holds for $m=s-1$.

Let us check (i) for $m=s$. Recall that $M(\lambda)_{\mu}^{k}=0$ for $k>>0$ and so $a_{k, j}=0$ for $k>>j$. Notice that $a_{k, 0}=0$ for $k \geq 1$; this gives

$$
\sum_{k \geq 1} \operatorname{ch}_{>\lambda-s \delta} M(\lambda)^{k}=\sum_{k, j \geq 1} a_{k, j} \operatorname{ch}_{>\lambda-s \delta} L(\lambda-j \delta)=\sum_{k, j \geq 1} a_{k, j} e^{-j \delta} \operatorname{ch}_{>\lambda-(s-j) \delta} L(\lambda) .
$$

Using the induction hypothesis we get

$$
e^{-\lambda} \sum_{k \geq 1} \operatorname{ch}_{>\lambda-s \delta} M(\lambda)^{k}=\sum_{k, j \geq 1} a_{k, j} e^{-j \delta} P_{>-(s-j) \delta}(Z) .
$$

On the other hand, the formula (8) gives

$$
e^{-\lambda} \sum_{k \geq 1} \operatorname{ch}_{>\lambda-s \delta} M(\lambda)^{k}=P_{>-s \delta}\left(Z \sum_{k \geq 1} \operatorname{ch} \mathcal{S}^{\geq k}\right)=\sum_{k, j \geq 1} \operatorname{dim} \mathcal{S}_{-j \delta}^{\geq k} e^{-j \delta} P_{>-(s-j) \delta}(Z) .
$$

Hence

$$
\sum_{k, j \geq 1} b_{k, j} e^{-j \delta} P_{>-(s-j) \delta}(Z)=0, \text { where } b_{k, j}:=a_{k, j}-\operatorname{dim} \mathcal{S}_{-j \delta}^{\geq k} .
$$

By $3.2(\mathrm{~A}) b_{k, j} \geq 0$ for all $k, j$.

Note that $a_{k, j}=\mathcal{S}_{-j \delta}^{\geq k}=0$ for $k>>j$. Observe that the term $P_{>-(s-j) \delta}(Z)=0$ iff $j \geq s$; for $j=1, \ldots, s-1$ this term is a finite sum of the form $\sum_{i} c_{i} e^{\mu_{i}}$, where $c_{i} \geq 0$. Hence the sum in the left-hand side of (15) is finite. Now combining the above observation and the inequalities $b_{k, j} \geq 0$, we get $b_{k, j}=0$ for $j=1, \ldots, s-1$. Hence $a_{k, j}=\operatorname{dim} \mathcal{S}_{-j \delta}^{\geq k}$ for $j=1, \ldots, s-1$. Since $a_{k, 0}=\delta_{k, 0}=\operatorname{dim} \mathcal{S}_{-j \delta}^{\geq k}$ we obtain (i) for $m=s$.

8.2.3. For (ii) write

$$
\operatorname{ch}_{>\lambda-s \delta} M(\lambda)=\operatorname{ch}_{>\lambda-s \delta} L(\lambda)+\sum_{j=1}^{s-1} a_{1, j} \operatorname{ch}_{>\lambda-s \delta} L(\lambda-j \delta) .
$$

Using (i) we obtain

$$
\begin{aligned}
\operatorname{ch}_{>\lambda-s \delta} L(\lambda) & =\operatorname{ch}_{>\lambda-s \delta} M(\lambda)-\sum_{j=1}^{s-1} \operatorname{dim} \mathcal{S}_{-j \delta} \operatorname{ch}_{>\lambda-s \delta} L(\lambda-j \delta) \\
& =e^{\lambda} P_{>-s \delta}(\operatorname{ch} \mathcal{S} \cdot Z)-\sum_{j=1}^{s-1} \operatorname{dim} \mathcal{S}_{-j \delta} e^{-j \delta} \operatorname{ch}_{>\lambda-(s-j) \delta} L(\lambda) .
\end{aligned}
$$


Finally, by the induction hypothesis,

$$
e^{-\lambda} \operatorname{ch}_{>\lambda-s \delta} L(\lambda)=P_{>-s \delta}(\operatorname{ch} \mathcal{S} \cdot Z)-\sum_{j=1}^{s-1} \operatorname{dim} \mathcal{S}_{-j \delta} e^{-j \delta} P_{>-(s-j) \delta}(Z)=P_{>-s \delta}(Z),
$$

and this establishes (ii).

\section{VANishing LEMMAS}

9.1. The following lemma is proven in [GS], 8.1.

Lemma. Let $\mathfrak{m}$ be a subalgebra of $\hat{\mathfrak{n}}$. Assume that $\lambda, \mu \in \hat{\mathfrak{h}}^{*}$ are such that

$$
L(\lambda)_{\mu} \subset \sigma(\mathfrak{q}) L(\lambda), \quad M^{\prime}(\lambda)_{\mu} \subset \sigma(\mathfrak{m}) M^{\prime}(\lambda)
$$

then

$$
H^{r}(\mathfrak{m}, L(\lambda))_{\mu}=0 \text { for } r=0,1 .
$$

9.2. Let $\mathfrak{m}$ be a Lie superalgebra which carries a $\mathbb{Z}_{\geq 0}^{k}$-grading $\mathfrak{m}=\oplus_{\nu \in \mathbb{Z}_{\geq 0}^{k}} \mathfrak{m}_{\nu}$ with finitedimensional homogeneous components. Set

$$
R:=\mathbb{C}[x], \quad A:=\mathbb{C}[x]_{(x)},
$$

that is $A$ is the localization of $R$ by the ideal generated by $x$.

Set $\mathfrak{m}_{R}:=\mathfrak{m} \otimes_{\mathbb{C}} R$. Let $M=\oplus_{\nu \in \mathbb{Z}_{<0}^{k}} M_{\nu}$ be a graded $\mathfrak{m}_{R}$-module whose homogeneous components $M_{\nu}$ are free $R$-modules of finite rank.

For each $c \in \mathbb{C}$ view $M(c):=M /(t-c) M$ as an $\mathfrak{m}$-module. Set $\mathfrak{m}_{A}:=\mathfrak{m} \otimes_{R} A$, $M_{A}:=M \otimes_{R} A$ and view $M_{A}$ as an $\mathfrak{m}_{A}$-module. In the following lemma $\mathfrak{m}_{A}$ is considered as a Lie superalgebra over $A$.

\subsubsection{Lemma. One has}

(i) $H^{0}(\mathfrak{m}, M(0))_{\nu}=0 \Longrightarrow H^{0}\left(\mathfrak{m}_{A}, M_{A}\right)_{\nu}=0$.

(ii) Suppose that $H^{0}(\mathfrak{m}, M(c))_{\nu}=H^{1}(\mathfrak{m}, M(c))_{\nu}=0$ for $c \in\{0\} \cup S$, where $S$ is an infinite subset of $\mathbb{C}$. Then $H^{1}\left(\mathfrak{m}_{A}, M_{A}\right)_{\nu}=0$.

Proof. (i) If $H^{0}\left(\mathfrak{m}_{A}, M\right)_{\nu} \neq 0$ then there exists $v \in M_{\nu}$ such that $\mathfrak{m} v=0$. Write $v=t^{k} w$ for $w \notin(t) M$. Then $\mathfrak{m} w=0$ and the image of $w$ in $M(0)=M /(t) M$ is non-zero; this contradicts to $H^{0}(\mathfrak{m}, M(0))_{\nu}=0$.

(ii) Let $\phi: \mathfrak{m}_{A} \rightarrow M_{A}$ be an $A$-linear map satisfying the conditions $\phi\left(\mathfrak{m}_{A, \mu}\right)=M_{\mu+\nu}$ and $\phi\left(\left[u_{1}, u_{2}\right]\right)=u_{1} \phi\left(u_{2}\right)-(-1)^{p\left(u_{1}\right) p\left(u_{2}\right)} u_{2} \phi\left(u_{1}\right)$. The required equality $H^{1}\left(\mathfrak{m}_{A}, M_{A}\right)_{\nu}=0$ means that $M_{A, \nu}$ contains a vector $v$ such that $u v=\phi(u)$ for any $u \in \mathfrak{m}$. 
Observe that $\mathfrak{m}_{A, \mu} M_{A, \nu}=0$ and $\phi\left(\mathfrak{m}_{A, \mu}\right)=0$ if $-(\mu+\nu) \notin \mathbb{Z}_{\geq 0}^{k}$ and that $\sum_{\mu:-(\mu+\nu) \in \mathbb{Z}_{\geq 0}^{k}} \mathfrak{m}_{A, \mu}$ has a finite rank over $A$. Let $k$ be the rank of $M_{\nu}$ over $R$ and $B$ be a basis of $M_{\nu}$. Write $v=\sum_{b \in B} x_{b} b$ with $x_{b} \in A$. The condition $u v=\phi(u)$ for all $u \in \mathfrak{m}$ can be written as a finite system of linear equations on $\left\{x_{b}\right\}_{b \in B}$ (the finiteness follows from the above observation), i.e. $D X=Y$, where $X=\left(x_{b}\right)_{b \in B}$ and $D$ is a matrix, $Y$ is a vector with entries in $A$. Let $S_{1} \subset S \cup\{0\}$ consist of $c$ 's such that the entries of $D$ and $Y$ have no poles at c. Note that $S_{1}$ is infinite and contains 0 .

For $c \in S_{1}$ let $D(c), Y(c)$ be the evaluations of respectively $D$ and $Y$ at $t=c$. We claim that the system $D(c) X^{\prime}=Y(c)$ has a unique solution for any $c \in S_{1}$, that is

$$
\forall c \in S_{1} \quad \exists ! X^{\prime} \in \mathbb{C}^{k} \text { s.t. } D(c) X^{\prime}=Y(c) .
$$

By Lemma 9.2.2, (16) implies that $D X=Y$ has a unique solution and so establishes (ii).

It remains to verify (16). For $c \in S_{1}$ let $\phi(c): \mathfrak{m} \rightarrow M(c)$ be the evaluation of $\phi$ at $t=c$. The assumption $H^{0}(\mathfrak{m}, M(c))_{\nu}=H^{1}(\mathfrak{m}, M(c))_{\nu}=0$ means that there exists a unique vector $w \in M(c)_{\nu}$ such that $\phi(c)(u)=u w$ for all $u \in \mathfrak{m}$. Let $B(c)$ be the image $B$ in $M(c)$. Then $w=\sum_{b^{\prime} \in B(c)} x_{b^{\prime}}^{\prime} b^{\prime}$ with $x_{b^{\prime}}^{\prime} \in \mathbb{C}$, where $X^{\prime}=\left(x_{b^{\prime}}^{\prime}\right)_{b^{\prime} \in B(c)}$ satisfies the system $D(c) X^{\prime}=Y(c)$ and this establishes (16) and completes the proof.

9.2.2. Set $F:=$ Fract $\mathbb{C}[x]$. For a matrix $D$ over $F$ and $c \in \mathbb{C}$ we say that " $D$ has no poles at $t=c$ " if the entries of $D$ have no poles at $c$; in this case we denote by $D(c)$ its evaluation at $c \in \mathbb{C}$.

Lemma. Take $D \in \operatorname{Mat}_{m \times k}(F), Y \in F^{m}$. Assume that there exists an infinite set $S \subset \mathbb{C}$ such that $D, Y$ have no poles in $S$ and

$$
\forall c \in S \quad \exists ! X^{\prime} \in \mathbb{C}^{k} \text { s.t. } D(c) X^{\prime}=Y(c) \text {. }
$$

Then there exists a unique $X \in F^{k}$ such that $D X=Y$; moreover, $X$ has no poles in $S$.

Proof. Fix $s \in S$. Since $D(s) X^{\prime}=Y(s)$ has a unique solution, $D(s)$ has a square submatrix $C^{\prime}$ of size $k$ such that $\operatorname{det} C^{\prime} \neq 0$. Let $C$ be the corresponding submatrix of $D$ and $Z$ be the corresponding sub-column of $Y$ (i.e. $Z$ is the transpose of $\left(y_{i_{1}}, \ldots, y_{i_{k}}\right)$, where $\left.C=\left(d_{i j}\right)_{i_{1}, \ldots, i_{k} ; j=1, \ldots, k}\right)$. Clearly, $C^{\prime}=C(s)$, and $(\operatorname{det} C)(s)=\operatorname{det} C(s)$ so $(\operatorname{det} C)(s) \neq 0$. In particular, $\operatorname{det} C \neq 0$ so $C$ is invertible and $D X=Y$ has at most one solution.

Let us show that $D X=Y$ for $X:=C^{-1} Z$ and that $X$ has no poles in $S$.

Let $S_{1}:=\{c \in S \mid(\operatorname{det} C)(c) \neq 0\}$. Note that $S_{1}$ is infinite and contains $s ; X$ has no poles in $S_{1}$. Take $c \in S_{1}$. Then $\operatorname{det} C(c) \neq 0$ so $C(c)$ is a non-zero minor of the maximal size in the system $D(c) X^{\prime}=Y(c)$. Since this system has a unique solution, this solution is $C(c)^{-1} Z(c)=X(c)$. Hence $D(c) X(c)=Y(c)$ for any $c \in S_{1}$. Since $S_{1}$ is infinite and the entries of $D(c), X(c)$ and $Y(c)$ are rational functions in $c$, we conclude that $D X=Y$. Hence a unique solution of the system $D X=Y$ has no poles at $s$. Since $s$ is an arbitrary element of $S$ this solution has no poles in $S$. 


\section{REFERENCES}

[Ar] T. Arakawa, A new proof of the Kac-Kazhdan conjecture, to appear in IMRN.

[DGK] V. V. Deodhar, O. Gabber, V. G. Kac, Structure of some categories of representations of infinite dimensional Lie algebras, Adv. in Math., 45, (1982), 92-116.

[Ch] V. Chari, Integrable representation of affine Lie algebras, Inv. Math., 85 (1986), 317-335.

[H] T. Hayashi, Sugawara operators and Kac-Kazhdan conjecture, Inv. Math., 94 (1988), 13-52.

[FF] B. Feigin, E. Frenkel, The family of representations of affine Lie algebras, Russian Mth. Survey, 43, (1988), 221-222.

[F] E. Frenkel, Wakimoto modules, opers and the center at the critical level, Adv. in Math., 195, (2005) no. 2, 297-404.

[GW] R. Goodman, N. Wallach, Higher order Sugawara operators for affine Kac-Moody algebras, Trans. AMS, 315, (1989), 1-66.

[GK] M. Gorelik, V. G. Kac, On simplicity of vacuum modules, arXiv: math-ph/0606002.

[GS] M. Gorelik, V. Serganova, Verma modules over $Q(n)^{(2)}$, preprint.

[HS] C. Hoyt, V. Serganova, Classification of finite-growth general Kac-Moody superalgebras, to appear in Comm. in Algebra.

[Ja] J. C. Jantzen, Moduln mit einem höchsten Gewicht, Lecture Notes in Math., $750,1979$.

[K1] V. G. Kac, Lie Superalgebras, Advances in Math. 26, (1977), 8-96.

[K2] V. G. Kac, Infinite Dimensional Lie Algebras, Progress in Math. 44, Birkhäuser, Boston, 1983.

[KK] V. G. Kac, D. A. Kazhdan, Structure of representations with highest weight of infinite dimensional Lie algebra, Adv. in Math., 34, (1979), 97-108.

$[\mathrm{KW}] \quad$ V. G. Kac, M. Wakimoto, Integrable highest weight modules over affine superalgebras and number theory, in Lie theory and geometry, 415-456, Progr. Math. 123 (1994) arXiv: hep-th/9407057.

[Ku] J.-M. Ku, Structure of the Verma module $M(-\rho)$ over Euclidean Lie algebras, J. of Algebra, 124, (1989), 367-387.

[M] O. Mathieu, On some modular representations of affine Kac-Moody algebras at the critical level, 102, (1996), 305-312.

[PS] I. Penkov, V. Serganova, Generic irreducible representations of finite-dimensional Lie superalgebras, International Journal of Math., 5 (1994), 389-419.

[S] V. Serganova, Automorphisms of simple Lie superalgebras, Math. USSR Izv., 24 (1985), $539-551$.

[Sz] M. Szczesny, Wakimoto modules for twisted affine Lie algebras, Math. Res. Lett., 9, (2002), 433-448.

[vdL] J. W. van de Leur, A classification of contragredient Lie superalgebras of finite growth, Comm. in Algebra, 17, (1989), 1815-1841.

[Wk1] M. Wakimoto, Fock reprsentations of the affine Lie algebra $A_{1}^{(1)}$, Comm. Math. Phys., 104, (1986), 604-609.

[Wk2] M. Wakimoto, Infinite Dimensional Lie Algebras, Translation of Mathematical Monographs 195 (2001).

[Wl] N. Wallach, A class of non-standard modules for affine Lie algebras, Math. Z. 196, (1987) No. 3, 303-313.

email: maria.gorelik@weizmann.ac.il 